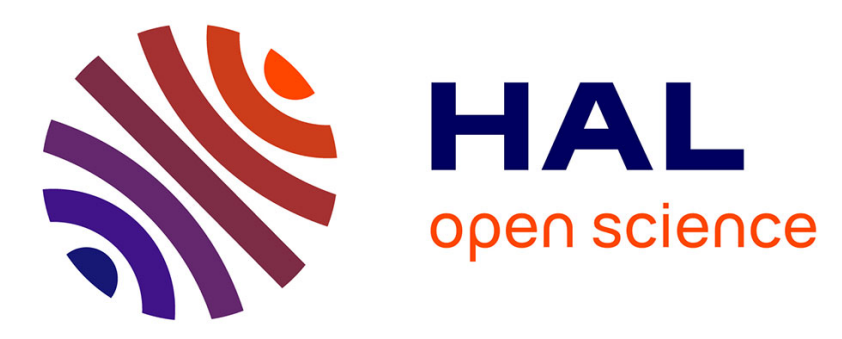

\title{
A graph theory approach to identify resonant and non-resonant transmission paths in statistical modal energy distribution analysis
}

Àngels Aragonès, Laurent Maxit, Oriol Guasch

\section{To cite this version:}

Àngels Aragonès, Laurent Maxit, Oriol Guasch. A graph theory approach to identify resonant and non-resonant transmission paths in statistical modal energy distribution analysis. Journal of Sound and Vibration, 2015, pp.91-110. 10.1016/j.jsv.2015.04.001 . hal-01162151

\section{HAL Id: hal-01162151 \\ https://hal.science/hal-01162151}

Submitted on 9 Jun 2015

HAL is a multi-disciplinary open access archive for the deposit and dissemination of scientific research documents, whether they are published or not. The documents may come from teaching and research institutions in France or abroad, or from public or private research centers.
L'archive ouverte pluridisciplinaire $\mathbf{H A L}$, est destinée au dépôt et à la diffusion de documents scientifiques de niveau recherche, publiés ou non, émanant des établissements d'enseignement et de recherche français ou étrangers, des laboratoires publics ou privés. 


\title{
A graph theory approach to identify resonant and non-resonant transmission paths in statistical modal energy distribution analysis
}

\author{
Àngels Aragonès ${ }^{\mathrm{a}}$, Laurent Maxit ${ }^{\mathrm{b}}$, Oriol Guasch ${ }^{\mathrm{a}}$ \\ ${ }^{a}$ GTM - Grup de recerca en Tecnologies Mèdia, La Salle, Universitat Ramon Llull \\ C/ Quatre Camins 2, 08022 Barcelona, Catalonia \\ ${ }^{b}$ INSA-Lyon, Laboratoire Vibrations-Acoustique (LVA), \\ 25 bis, av. Jean Capelle, F-69621, Villeurbanne Cedex, France
}

\section{Abstract}

Statistical modal energy distribution analysis (SmEdA) extends classical statistical energy analysis (SEA) to the mid frequency range by establishing power balance equations between modes in different subsystems. This circumvents the SEA requirement of modal energy equipartition and enables applying SmEdA to the cases of low modal overlap, locally excited subsystems and to deal with complex heterogeneous subsystems as well. Yet, widening the range of application of SEA is done at a price with large models because the number of modes per subsystem can become considerable when the frequency increases. Therefore, it would be worthwhile to have at one's disposal tools for a quick identification and ranking of the resonant and nonresonant paths involved in modal energy transmission between subsystems. It will be shown that previously developed graph theory algorithms for transmission path analysis (TPA) in SEA can be adapted to SmEdA and prove useful for that purpose. The case of airborne transmission between two cavities separated apart by homogeneous and ribbed plates will be first addressed to illustrate the potential of the graph approach. A more complex case representing transmission between non-contiguous cavities in a shipbuilding structure will be also presented.

Keywords: Statistical modal energy distribution analysis, Graph theory, Transmission path analysis

\section{Introduction}

The mid-frequency problem in the vibration analysis of built-up mechanical structures appears when neither low frequency deterministic approaches (e.g., finite elements, semi-analytical modal expansions) nor high frequency statistical energy methods (e.g., statistical energy analysis, SEA) can be used for the correct description of the system response. Inconveniences with the former are due to the increase in computational cost with frequency and to the fact that the vibrational behavior becomes strongly sensitive to the variability in the structure properties. Yet, energy methods based on averaged power balance equations like SEA usually require the assumption of some strong hypotheses concerning, for instance, the modal overlap and modal number of the subsystems into which the structure has been divided (see e.g., [1]).

In recent years, several strategies have been followed to tackle the mid-frequency problem. Some approaches have attempted at extending the range of applicability of deterministic methods to higher frequencies. For instance, this is the case of wave based methods (WBM) which adopt exact solutions of the governing partial differential equations as basis functions, instead of the polynomials bases of finite elements (FEM), see e.g., [2] for a review. Another option could be the scaling procedure proposed in the asymptotical scaled modal analysis (ASMA) in [3]. Other methods tried combining deterministic and statistical methods. In [4], a scale separation approach was proposed in which large scales were resolved using a deterministic modal approach whereas the influence of small scales was taken into account using SEA. The coexistence of deterministic and statistical subsystems for a given frequency band, typical of aircraft and submarine hulls, has been addressed by means of hybrid approaches that couple FEM with SEA [5-7]. In [8, 9], a hybrid approach was also proposed using power flow analysis and SEA. 
SEA has also been derived as limiting cases of wave intensity analysis and travelling wave packets [1012], and recently presented as a low resolution ray-tracing technique [13]. Besides, SEA can alternatively be viewed as a particular case of the more general energy distribution (ED) models (also referred to as energy flow models), which are likewise based on averaged power flow equations and where energy transmission is characterized by means of the so called energy influence coefficients [14]. What conditions an ED model should satisfy to become an SEA model has been a matter of research [15] leading to the definition of quasi and proper SEA systems $[16,17]$.

Statistical modal energy distribution analysis (SmEdA), originally proposed in [18-20], can be envisaged in this last group of ED methods, in which SEA hypotheses are relaxed to extend SEA's range of applicability to mid frequencies. However, SmEdA has a clear distinctive feature with respect to most ED methods in the sense that power balance equations are not established between subsystems but rather between the resonant modes of different subsystems. These modes can be extracted from the modal bases of uncoupled subsystems, which can be computed using FEM, thanks to the dual modal formulation (DMF) [19]. This offers the possibility of considering subsystems with complex geometries and varying properties. Moreover, circumventing SEA energy equipartition allows one to deal with locally excited subsystems with low modal overlap [20], as well as to evaluate the spatial distribution of energy density within subsystems [21]. Recently, SmEdA has been extended to incorporate the contribution of non-resonant transmission through condensation of the DMF equations. This has resulted in the appearance of indirect coupling between modes in non-physically connected subsystems, standard non-resonant paths in SEA being recovered as a particular case $[22]$.

Though SmEdA may offer several advantages when compared to SEA, the price to be paid is that of dealing with large matrix systems (yet much smaller than those encountered in deterministic methods like FEM, which can involve millions of degrees of freedom). This makes the analysis of the obtained results difficult. For instance, determining which modes play a predominant role in the energy transmission between subsystems for even simple cases, such as two cavities separated by a homogeneous wall, may implicate hundreds of modes at mid-frequencies. A thorough analysis of the interaction of modal works and involved modal coupling loss factors then becomes necessary to find the dominant modal energy transmission paths between the excited cavity and the receiver one [22]. It is the goal of this work to try to lighten this process by resorting to an alternative approach. In particular, it will be shown that graph theory may prove useful for that purpose. The idea of using graph theory tools to solve vibroacoustics problems was first introduced in [23], where it was pointed out that it was possible to define a SEA graph, whose weighting matrix was identified with the generating matrix of the SEA system solution geometric series expansion. In [24], an adaptation of the MSP algorithm (see [25, 26]) for the efficient computation and ranking of energy transmission paths from sources to receivers in SEA systems was presented, the inclusion of variance recently being taken into account in [27]. Graph cut algorithms were also used in [28] in an optimizing process to diminish energy transmission between subsystems in SEA. The prospect of defining SEA graphs for the more general ED models has recently been deemed viable in [29]. In this work, it will be shown that this is also feasible for SmEdA models, so that path graph algorithms can be applied to identify and rank the relevant modes governing the energy transmission between subsystems.

The paper is organized as follows. In section 2 a brief overview on the basics of SmEdA is presented. In section 3 it is exposed how to define a SmEdA graph, and the notion of modal energy transmission paths becomes introduced. Two benchmark examples involving resonant and non-resonant energy transmission between two adjacent cavities separated apart by homogeneous and ribbed plates are presented in section 4 . It is shown how the graph theory approach can provide very valuable information in a quicker and more efficient way than when attempting a conventional analysis of the SmEdA results. A more complex case dealing with vibrational and acoustic energy transmission in a shipbuilding built-up structure is addressed in section 5. Conclusions close the paper in section 6 . 


\section{An overview of SmEdA}

\subsection{Resonant transmission in a two-subsystem SmEdA model}

An introduction to SmEdA will be next presented to describe its general performance, as well as to highlight some of its features which will allow one to establish a connection with graph theory. For the sake of simplicity, the SmEdA modal energy equations for a system simply made of two coupled subsystems will be first addressed.

As for SEA, the prerequisites of weak coupling and of dominant reverberant field will be assumed, so that the vibratory behavior of the two coupled subsystems can be solely described in terms of the energy transmission between resonant modes (i.e., modes having their modal frequencies within the frequency band of excitation). Numerical tests $[19,30]$ have shown that this assumption is compliant if the substructuring into subsystems is done well along mechanical impedance mismatch and if the boundary conditions at the coupling surfaces are correctly imposed to determine the subsystem modes. This can be achieved by describing the stiff subsystem by its uncoupled free modes (i.e., assuming free displacements on the coupling surface) while describing the soft one by its uncoupled blocked modes (i.e., imposition of null displacements on the coupling surface).

For illustrative purposes, consider that the two subsystems consist of an acoustic cavity (subsystem 1) coupled to a vibrating structure (subsystem 2). The cavity is therefore the soft subsystem and becomes characterized by means of its blocked modes (i.e., its boundary is assumed to be composed of rigid walls) whereas the vibrating structure is the stiff subsystem and its free modes (i.e. in vacuo modes) should be used instead. According to the dual modal formulation (see [18] for the case of two coupled mechanical subsystems and [22] for a cavity-structure problem), the acoustic pressure field (stress field in a general case) is the appropriate one to describe subsystem 1, whereas the displacement field is adequate for subsystem 2 . Denoting by $\hat{P}$ and $\hat{Q}$ the sets of resonant modes in subsystems 1 and 2 , respectively containing $N_{p}$ and $N_{q}$ modes, the acoustic pressure at point $M$ in the cavity and the displacement at point $N$ on the structure can be estimated using the modal expansions

$$
\begin{aligned}
& p(M, t)=\sum_{p \in \hat{P}} \xi_{p}(t) \tilde{p}_{p}(M), \\
& u(N, t)=\sum_{q \in \hat{Q}} \zeta_{q}(t) \tilde{u}_{q}(N),
\end{aligned}
$$

where $\xi_{p}$ and $\zeta_{q}$ denote modal amplitudes, $\tilde{p}_{p}$ stands for the spatial acoustic pressure distribution of the $p$-th cavity mode, and $\tilde{u}_{q}$ represents the displacement spatial shape of the $q$-th structure mode.

Following the DMF approach, expressions (1a)-(1b) are to be introduced in the weak formulation of the coupled problem. Taking advantage of the orthogonality of the uncoupled modes, presuming viscous damping and making the change of variables $\xi_{p}=\chi_{p}^{\prime}, \forall p \in \hat{P}$ (with the prime symbol indicating time derivative), the following modal equations of motion can be derived (see [18] for details)

$$
\begin{array}{ll}
\chi_{p}^{\prime \prime}(t)+\omega_{p} \eta_{p} \chi_{p}^{\prime}(t)+\omega_{p}^{2} \chi_{p}(t)-\sum_{q \in \hat{Q}} W_{p q} \zeta_{q}^{\prime}(t)=Q_{p}(t), & \forall p \in \hat{P}, \\
\zeta_{q}^{\prime \prime}(t)+\zeta_{q} \eta_{q} \zeta_{q}^{\prime}(t)+\omega_{q}^{2} \zeta_{q}(t)-\sum_{p \in \hat{P}} W_{p q} \chi_{p}^{\prime}(t)=0, & \forall q \in \hat{Q} .
\end{array}
$$

In (2a)-(2b), $\omega_{p}, \omega_{q}$ denote the modal angular frequencies and $\eta_{p}, \eta_{q}$ the modal damping loss factors. The mode shapes are supposed to be normalized to a unit modal mass for the free subsystem and to a unit modal stiffness for the blocked subsystem. $Q_{p}$ represents the modal source strength at mode $p$ due to external excitation and $W_{p q}$ corresponds to the modal interaction work between $p$ and $q$. For each pair of modes, the latter is defined as the integral over the coupling surface, $S_{C}$, of the product between a displacement mode shape of the free subsystem and a stress mode shape of the blocked subsystem. For the cavity-structure example the modal interaction work would be $W_{p q}=\int_{S_{C}} \tilde{p}_{p} \tilde{u}_{q} d S$. 


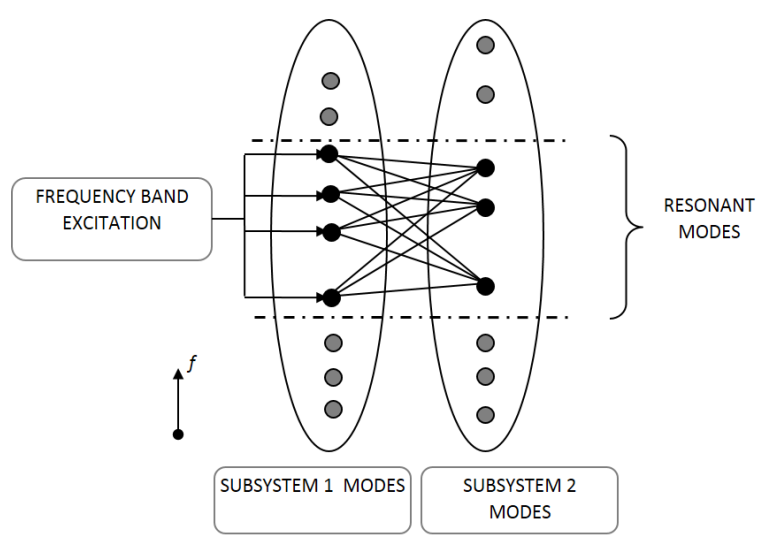

Figure 1: Sketch for the the modal coupling between two coupled subsystems.

The form of equations (2a)-(2b) allows one to interpret mode interactions as oscillators coupled by gyroscopic elements (which introduce opposite sign coupling forces proportional to the oscillator velocities [18]). A schematic representation of this modal coupling is proposed in Fig. 1. Note that a mode in one subsystem is coupled to all modes in the other subsystem but it is not coupled with the modes in its own subsystem. The number of back and forth modal energy direct couplings (the former symbolized with black lines in Fig. 1) is $2 N_{P} N_{Q}$, which strongly increases with the number of modes considered in each subsystem. Moreover, energy may obviously go back and forth between subsystem modes resulting in transmission paths of high order, the total number of them being infinite. In Section 3 it will be shown how to identify the ones dominating energy transmission in an efficient way.

SmEdA equations describe the power balance between the modes in different subsystems and are obtained from the principle of energy conservation for each mode in a subsystem. For the $p$-th mode in subsystem 1, they result in

$$
\Pi_{i n j}^{p}=\Pi_{d i s s}^{p}+\sum_{q \in \hat{Q}} \Pi_{p q}, \quad \forall p \in \hat{P}
$$

where $\Pi_{i n j}^{p}$ is the time-averaged injected power by the generalized force $Q_{p}, \Pi_{\text {diss }}^{p}$ is the time-averaged power dissipated by the internal damping of mode $p$ and $\Pi_{p q}$ is the time-averaged power flow exchanged between the resonant mode $p$ of subsystem 1 and the resonant mode $q$ of subsystem 2 . The various powers appearing in (3) can be evaluated from already established relations for one single oscillator and/or two coupled oscillators, using the same assumptions as in SEA (e.g., white noise force spectra and uncorrelated modal interaction forces [31]). It follows that

$$
\Pi_{i n j}^{p} \approx \frac{\pi}{4} \bar{S}_{Q_{p}}, \quad \Pi_{\text {diss }}^{p} \approx \omega_{p} \eta_{p} E_{p}, \quad \Pi_{p q} \approx \beta_{p q}\left(E_{p}-E_{q}\right)
$$

where $\bar{S}_{Q_{p}}$ is the power spectral density of the modal source strength, $E_{p}$ is the time averaged energy of mode $p$, and $\beta_{p q}$ is called the modal coupling factor given by (see [20]),

$$
\beta_{p q}=W_{p q}^{2}\left[\frac{\omega_{p} \eta_{p} \omega_{q}^{2}+\omega_{q} \eta_{q} \omega_{p}^{2}}{\left(\omega_{p}^{2}-\omega_{q}^{2}\right)^{2}+\left(\omega_{p} \eta_{p}+\omega_{q} \eta_{q}\right)\left(\omega_{p} \eta_{p} \omega_{q}^{2}+\omega_{q} \eta_{q} \omega_{p}^{2}\right)}\right] .
$$

Note that $\beta_{p q} \geq 0$ which will be crucial for the proper definition of modal energy transmission paths and SmEdA graphs in the following section.

The power balance equation for any resonant mode of subsystem 2 will be analogous to that of subsystem 1 in (3) (though for simplicity it will be supposed that no external force is acting on subsystem 2). The $N_{p}+N_{q}$ equations for all modes in subsystems 1 and 2 can be combined in the linear matrix system

$$
\left(\begin{array}{cc}
\beta_{11} & -\beta_{12} \\
-\beta_{12} & \beta_{22}
\end{array}\right)\left(\begin{array}{c}
E_{1} \\
E_{2}
\end{array}\right)=\left(\begin{array}{c}
\Pi_{1} \\
0
\end{array}\right)
$$


with unknown modal energies $\boldsymbol{E}_{\mathbf{1}}=\left(E_{p}\right)_{N_{P} \times 1}$ and $\boldsymbol{E}_{\mathbf{2}}=\left(E_{q}\right)_{N_{Q} \times 1}$, external input power at subsystem 1 modes $\boldsymbol{\Pi}_{\mathbf{1}}=\left(\Pi_{i n j}^{p}\right)_{N_{P} \times 1}$, coupling loss factor matrix $\boldsymbol{\beta}_{\mathbf{1 2}}=\left(\beta_{p q}\right)_{N_{P} \times N_{Q}}$ and diagonal loss factor matrices $\boldsymbol{\beta}_{\mathbf{1 1}}=\operatorname{diag}\left(\omega_{\mathrm{p}} \eta_{\mathrm{p}}+\sum_{\mathrm{q} \in \hat{\mathrm{Q}}} \beta_{\mathrm{pq}}\right)_{\mathrm{N}_{\mathrm{P} \times \mathrm{N}_{\mathrm{P}}}}$ and $\boldsymbol{\beta}_{\mathbf{2 2}}=\operatorname{diag}\left(\omega_{\mathrm{q}} \eta_{\mathrm{q}}+\sum_{\mathrm{p} \in \hat{\mathrm{P}}} \beta_{\mathrm{pq}}\right)_{\mathrm{N}_{\mathrm{Q}} \times \mathrm{N}_{\mathrm{Q}}}$. Inversion of (6) results in the SmEdA system modal energies. From the summation of all modal energies in a subsystem its overall energy can be obtained and then related to a spatial mean square velocity in the case of a vibrating plate, or to a mean square acoustic pressure in the case of a cavity, following standard SEA formulations [31-33].

\subsection{Resonant and non-resonant transmission in a three-subsystem SmEdA model}

A slightly more involved case is next considered which consists of a system made of three subsystems. This will allow one to inspect how non-resonant transmission can be accounted for in SmEdA, a topic that has only been addressed very recently [22]. For the ease of exposition and without loss of generality, suppose that the system is made of two cavities separated by a panel. Subsystems 1 and 3 are identified with the cavities and subsystem 2 with the panel (this system will be re-encountered in the benchmark examples of Section 4). The sets of resonant modes for the cavities in the frequency range of interest will be denoted by $\hat{P}$ and $\hat{R}, \hat{Q}$ standing for the set of panel resonant modes. The SmEdA matrix formulation analogous to (6) if external input power is supplied to the first cavity, will be given by

$$
\left(\begin{array}{ccc}
\boldsymbol{\beta}_{11} & -\boldsymbol{\beta}_{12} & 0 \\
-\boldsymbol{\beta}_{12} & \boldsymbol{\beta}_{\mathbf{2 2}}^{\prime} & -\boldsymbol{\beta}_{\mathbf{2 3}} \\
\mathbf{0} & -\boldsymbol{\beta}_{23} & \boldsymbol{\beta}_{33}
\end{array}\right)\left(\begin{array}{l}
\boldsymbol{E}_{1} \\
\boldsymbol{E}_{\mathbf{2}} \\
\boldsymbol{E}_{3}
\end{array}\right)=\left(\begin{array}{c}
\Pi_{1} \\
0 \\
0
\end{array}\right),
$$

with $\boldsymbol{E}_{\mathbf{1}}, \boldsymbol{E}_{\mathbf{2}}$ and $\boldsymbol{E}_{\mathbf{3}}$ respectively standing for the modal energy vectors of the first cavity, panel and second cavity.

Two remarks should be made with regard to (7). First, the diagonal matrix $\boldsymbol{\beta}_{\mathbf{2} 2}^{\prime}$ is different from $\boldsymbol{\beta}_{\mathbf{2 2}}$ in (6) because it incorporates the coupling with subsystem 3. Its expression becomes

$$
\boldsymbol{\beta}_{\mathbf{2 2}}^{\prime}=\operatorname{diag}\left(\omega_{\mathrm{q}} \eta_{\mathrm{q}}+\sum_{\mathrm{p} \in \hat{\mathrm{P}}} \beta_{\mathrm{pq}}+\sum_{\mathrm{r} \in \hat{\mathrm{R}}} \beta_{\mathrm{qr}}\right)_{\mathrm{N}_{\mathrm{Q}} \times \mathrm{N}_{\mathrm{Q}}}
$$

Second, the null blocks $\mathbf{0}$ in (7) indicate that there is no direct coupling between the resonant modes of both cavities, given that they are not physically connected. However, it is well known that resonant transmission, as described by the standard SmEdA approach (7), cannot correctly represent the whole acoustic transmission through the panel below the critical frequency, which is governed by the mass law. To tackle with this problem it was proposed in [22] to include the panel non-resonant modes in the analysis. Although the frequencies of these non-resonant panel modes do not coincide with those of the cavity resonant modes, the modes are strongly coupled one to another because of spatial matching. If one incorporates nonresonant transmission in the DMF equations, it turns out that after matrix condensation the former can be accounted for by establishing a direct coupling between the modes of both cavities (even though not being physically connected). The coupling factors $\beta_{p r}$ between modes in $\hat{P}$ and $\hat{R}$ are characterized by spring connections rather than gyroscopic ones. The stiffness of the spring connection can be related to the intermodal works between the resonant cavity modes and the non-resonant panel modes (see [22] for details).

As a consequence, when non-resonant paths are considered (7) transforms to

$$
\left(\begin{array}{ccc}
\boldsymbol{\beta}_{11}^{\prime \prime} & -\boldsymbol{\beta}_{12} & -\boldsymbol{\beta}_{13} \\
-\boldsymbol{\beta}_{12} & \boldsymbol{\beta}_{\mathbf{2 2}}^{\prime} & -\boldsymbol{\beta}_{\mathbf{2 3}} \\
-\boldsymbol{\beta}_{\mathbf{1 3}} & -\boldsymbol{\beta}_{\mathbf{2 3}} & \boldsymbol{\beta}_{\mathbf{3 3}}^{\prime \prime}
\end{array}\right)\left(\begin{array}{c}
\boldsymbol{E}_{\mathbf{1}} \\
\boldsymbol{E}_{\mathbf{2}} \\
\boldsymbol{E}_{\mathbf{3}}
\end{array}\right)=\left(\begin{array}{c}
\Pi_{1} \\
0 \\
0
\end{array}\right),
$$

where the block $\boldsymbol{\beta}_{\mathbf{1 3}}=\left(\beta_{p r}\right)_{N_{P} \times N_{R}}$ is no longer zero. Note that the matrices $\boldsymbol{\beta}_{\mathbf{1 1}}^{\prime \prime}$ and $\boldsymbol{\beta}_{\mathbf{3} \mathbf{3}}^{\prime \prime}$ differ from $\boldsymbol{\beta}_{\mathbf{1 1}}$ and $\boldsymbol{\beta}_{\mathbf{3 3}}$ in (9) because they include the terms corresponding to the direct connections between cavities, 
characterized by $\boldsymbol{\beta}_{\mathbf{1 3}}$. Numerical validations on test cases [22] had shown the ability of this SmEdA approach to describe the non-resonant transmission through the panel, and in particular, the mass law behaviour. However, determining which cavity and panel modes play a relevant role in the transmission through spatial matching may be rather lengthy and intricate. A fast way to do so by resorting to graph theory will be presented for the cavity-panel-cavity system in Section 4.

\subsection{General matrix formulation for an $N$-subsystem SmEdA model}

Generalization of the above SmEdA matrix system formulation for a model consisting of $N$ subsystems that includes both, resonant and non-resonant transmission, is straightforward and yields

$$
\left(\begin{array}{ccccc}
\boldsymbol{\beta}_{11} & -\boldsymbol{\beta}_{12} & -\boldsymbol{\beta}_{13} & \cdots & -\boldsymbol{\beta}_{1 N} \\
-\boldsymbol{\beta}_{12}{ }^{\top} & \boldsymbol{\beta}_{22} & -\boldsymbol{\beta}_{23} & \cdots & -\boldsymbol{\beta}_{2 N} \\
-\boldsymbol{\beta}_{13}{ }^{\top} & -\boldsymbol{\beta}_{23}{ }^{\top} & \boldsymbol{\beta}_{33} & \cdots & -\boldsymbol{\beta}_{3 N} \\
\vdots & \vdots & \vdots & \ddots & \vdots \\
-\boldsymbol{\beta}_{1 N}{ }^{\top} & -\boldsymbol{\beta}_{2 N}{ }^{\top} & -\boldsymbol{\beta}_{3 N}{ }^{\top} & \cdots & \boldsymbol{\beta}_{N N}
\end{array}\right)\left(\begin{array}{c}
\boldsymbol{E}_{1} \\
\boldsymbol{E}_{2} \\
\boldsymbol{E}_{3} \\
\vdots \\
\boldsymbol{E}_{N}
\end{array}\right)=\left(\begin{array}{c}
\Pi_{1} \\
\Pi_{2} \\
\Pi_{3} \\
\vdots \\
\Pi_{N}
\end{array}\right),
$$

with $\boldsymbol{E}_{\boldsymbol{i}}=\left(E_{i}\right)_{I \times 1}$ standing for the vector of modal energies of the $i$-th subsystem where an external power $\boldsymbol{\Pi}_{\boldsymbol{i}}=\left(\Pi_{i n j}^{i}\right)_{I \times 1}$ is being input. In order to lighten forthcoming expressions, system (10) will be simply rewritten as

$$
\beta E=\Pi \text {. }
$$

\section{Modal energy transmission paths and SmEdA graphs}

\subsection{Modal energy transmission paths in $\operatorname{SmEdA}$}

The definition of energy transmission paths between subsystems in SEA models was introduced by Craik in $[32,34]$. As quoted in [35], the notion of transmission paths between two adjacent subsystems relies on the concept of blocked transmissibility [36-38]; the energy transmitted from an arbitrary subsystem $i$ to a neighboring subsystem $j$ is given by the quotient of energies $E_{j} / E_{i}$ when $i$ is excited and all energies in the SEA model but $i$ and $j$ are set to zero. This results in $E_{j} / E_{i}=\eta_{i j} / \eta_{j}, \eta_{i j}$ standing for the SEA coupling loss factor between $i$ and $j$, and $\eta_{j}$ for the total loss factor of subsystem $j$. Therefore $\eta_{i j} / \eta_{j}$ can be interpreted as the fraction of energy at $j$ that directly comes from $i$, and thus identified with the weight of a first order transmission path connecting subsystems $i$ and $j$ i.e., $w\left(p_{i j}^{1}\right)=\eta_{i j} / \eta_{j}$. A second order path $p_{s t}^{2}$ linking e.g., subsystem $s$ with $t$ through a third subsystem $j$ could be built concatenating the first order paths $p_{s j}^{1}$ and $p_{j t}^{1}$, with weight $w\left(p_{s t}^{2}\right)=\left(\eta_{s j} / \eta_{j}\right)\left(\eta_{j t} / \eta_{t}\right)$. Following this procedure arbitrary $n$-order paths linking $n+1$ subsystems can be built having weights $w\left(p_{s t}^{n}\right)=\Pi_{h_{i}=1}^{n-1}\left(\eta_{h_{i} h_{i+1}} / \eta_{h_{i}}\right)$ (s being identified with $h_{1}$ and $t$ with $\left.h_{n-1}\right)$, see $[23,24]$ for details.

The same line of reasoning can be pursued to define energy transmission in SmEdA, from an arbitrary mode $p$ in a subsystem $P$ to a mode $q$ in subsystem $Q$. The weight of the first order order transmission path, $p_{p q}^{1}$, from mode $p \in \hat{P}$ to $q \in \hat{Q}$, in the case of subsystems $P$ and $Q$ being adjacent, will be given by

$$
w\left(p_{p q}^{1}\right)=\frac{\beta_{p q}}{\beta_{q}}
$$

A general $n$-th order path between a mode $s \in \hat{S}$ and a mode $t \in \hat{T}$ in the SmEdA system can then be built by concatenation as

$$
w\left(p_{s t}^{n}\right)=\frac{\beta_{s h_{1}}}{\beta_{h_{1}}} \frac{\beta_{h_{1} h_{2}}}{\beta_{h_{2}}} \cdots \frac{\beta_{h_{n-1} t}}{\beta_{t}}
$$

Note from the considerations in Section 2.1 that (13) makes sense given that $\beta_{h_{i} h_{i+1}} \geq 0$ and $\beta_{h_{i}}>0$, $\forall h_{i} \in \hat{P}, h_{i+1} \in \hat{Q}$. Note also that, as specified, no transmission is allowed from a mode in one subsystem 
to another mode in the same subsystem. However, a path going from one mode, say $p_{1} \in \hat{P}$ to $q \in \hat{Q}$ and then back to $p_{2} \in \hat{P}$, or even $p_{1} \in \hat{P}$, is perfectly feasible.

A transmission path, as defined in (13), allows one to know the energy that has been transmitted from a particular source mode, where the external energy is input, to a target mode in a different subsystem, involving a particular set of intermediate modes. As it will be shown in Section 4, the fast and efficient computation of such paths will prove very useful to determine which modes play a significant role in resonant and non-resonant transmission in SmEdA models. However, it should be remarked that in order for transmission path analysis to be a well-posed problem from a mathematical and physical point of view, an additional condition has to be satisfied. It has to be possible to recover the overall energy at any target mode as the summation of the energy contributions of all transmission paths linking the source mode with the target one (see [24] for a detailed explanation in the case of SEA). Assuming fulfillment of that condition, a transmission path analysis makes sense from a practical point of view whenever the energy transmission is justified by a small set of dominant paths, so that one could act on their constituent modes/subsystems for remedial action.

Let us next see that transmission path analysis in SmEdA is in fact a well-posed problem. Define the matrix $\boldsymbol{B}:=\boldsymbol{I}-\operatorname{diag}\left(1 / \beta_{i i}\right) \boldsymbol{\beta}$ whose diagonal is null and its off-diagonal entries non-negative. This matrix is also irreducible, i.e. it cannot be made similar to a block upper triangular matrix via a permutation. This amounts to saying that the adjacency matrix associated to $\boldsymbol{B}$ is that of a strongly connected directed graph (this is actually the case, see next section). Next one can make use of the Perron-Frobenius theorem (see e.g., [39]) and easily show that the spectral radius of $\boldsymbol{B}, \rho(\boldsymbol{B})<1$. As a consequence $\boldsymbol{B}$ is convergent, i.e., the series of matrices $\boldsymbol{B}^{m}, m=0,1,2 \ldots$, converge to the null matrix. Defining $\boldsymbol{\Pi}^{\prime}=\operatorname{diag}\left(1 / \beta_{\mathrm{ii}}\right) \boldsymbol{\Pi}$ permits rewriting (11) as

$$
(\boldsymbol{I}-\boldsymbol{B}) \boldsymbol{E}=\boldsymbol{\Pi}^{\prime}
$$

so that

$$
\boldsymbol{E}=(\boldsymbol{I}-\boldsymbol{B})^{-1} \boldsymbol{\Pi}^{\prime}=\left(\sum_{n=0}^{\infty} \boldsymbol{B}^{n}\right) \boldsymbol{\Pi}^{\prime} .
$$

This proves that the energy at any mode can effectively be recovered as the infinite summation of the energy contributions of the transmission paths linking the SmEdA system source modes with the target ones. The entries in $\boldsymbol{B}^{\top}$ correspond to the energy contribution of the first order paths between pairs of modes; note that $B_{p q}=\beta_{p q} / \beta_{p}, p \neq q$, which according to (12) corresponds to a path from $q$ to $p$ (v. $\beta_{p q}=\beta_{q p}$ ). Thus $B_{q p}$ will be the entry for a path from $p$ to $q$. In the same way, the transposed of $\boldsymbol{B}^{n}$ includes the total contribution of the $n$-th order paths linking modes in the SmEdA system. As the paths get longer their contribution decreases because as said $n \rightarrow \infty \Rightarrow \boldsymbol{B}^{n} \rightarrow \mathbf{0}$. A series like (15) was first proposed for SEA systems in [40], see also [13, 23, 32, 40]. As will be shown next, (15) constitutes the key to link SmEdA with graph theory.

\subsection{The SmEdA graph}

Succinctly, a graph $G=(U, E)$ consists of two sets of elements, $U$ being the set of nodes and $E$ the set of arcs, or edges, connecting the nodes. The arcs in $E$ can be assigned numerical values which can be gathered in the so called weighting matrix of the graph [41]. A SmEdA graph can be built by defining all subsystem modes as the nodes of the graph and identifying the arcs with the first order paths connecting them. The arcs are then assigned the weights of these first order paths. Note that this would come down to identifying the transpose of the generating matrix in (13), $\boldsymbol{B}^{\top}$, as the weighting matrix of a strongly connected directed SmEdA graph, analogously to what is done in SEA [23, 24]. The graph is strongly connected because for any two arbitrary nodes in the graph there always exists a path connecting them.

For the correct definition of a SmEdA graph an additional subtle point has to be considered. In practice it is not possible to excite a particular mode of a given subsystem; standard mechanisms excite the whole subsystem at once. Moreover, one is usually interested in knowing the influence of a given transmission path to the whole target subsystem energy, not to one of its modes. To take into account these two facts, the SmEdA graph has to be enlarged with two fictitious nodes (not corresponding to system modes), one 


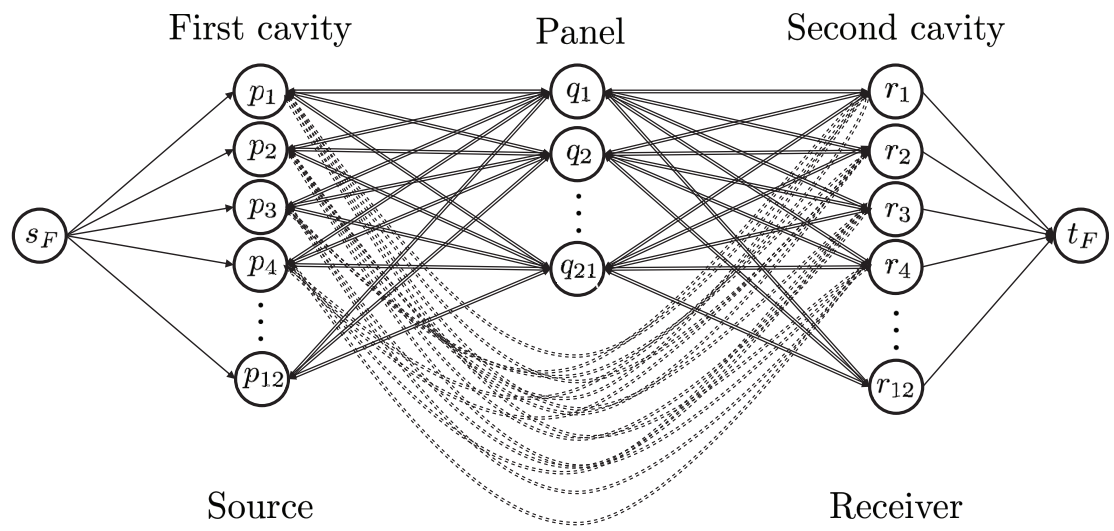

Figure 2: SmEdA graph corresponding to the cavity-panel-cavity model in Fig. 3

connected to the source subsystem modes, and the other one to the target subsystem modes. With regard to the former, one of the advantages of SmEdA is precisely that it can deal with both, localized and rain on the roof excitations. Let us denote the source subsystem as $P$ with modes $p \in \hat{P}$. A fictitious source node $s_{F}$ will be included in the graph with one-direction connections to every $p \in \hat{P}$. The weights of these connections will depend on the excitation being point-like or distributed. The power balance equation for a mode in the source subsystem $P$, when setting all remaining SmEdA system modal energies to zero, provides

$$
\Pi_{i n j}^{p}=\beta_{p} E_{p} .
$$

In the case of a rain on the roof excitation $\Pi_{i n j}^{p}$ will be constant for all $p \in \hat{P}$. As opposes to this, in the case e.g. of a monopole source term, $\Pi_{i n j}^{p} \approx(\pi / 4) \bar{S}_{Q_{p}}$, with $\bar{S}_{Q_{p}}$ standing for the power spectral density of the monopole, as stated in (4). The following weights will be thus assigned to the edges linking $s_{F}$ to every $p \in \hat{P}$,

$$
w_{s_{F} p}=\frac{\Pi_{i n j}^{p}}{\beta_{p}} .
$$

With regard to the target or receiver subsystem, say $R$, another fictitious node $t_{F}$ is included in the graph. All modes $r \in \hat{R}$ become connected to it through arcs with unitary weights $w_{r t_{F}}=1$. The energy at the target subsystem can be recovered from the summation of the energy of all its modes, $E_{T}=\sum_{r \in \hat{R}} E_{t}$.

To summarize, a SmEdA graph will consist of $(N+2)$ nodes, where $N$ is the total number of the SmEdA system modes. The two additional nodes are a fictitious source node $s_{F}$, which is connected to all the modes in the source subsystem with the weight in (17), and a fictitious target node to which all target subsystem modes become connected with unit weight. As an example of SmEdA graph, in Fig. 2 that corresponding to the cavity-panel-cavity example in Section 2.2 is presented, which will also be referred to in the forthcoming Section 4 (see also Fig. 3). The arcs plotted in solid lines correspond to resonant first order paths while the dashed line arcs correspond to non-resonant first order paths. The source is placed in the first cavity and the second cavity corresponds to the target subsystem. Therefore, the node $s_{F}$ is connected to all nodes in the first cavity, whereas all nodes in the second cavity become connected to $t_{F}$.

Once a SmEdA graph has been generated, use can be made of previously developed algorithms in graph theory for the computation of transmission paths linking the source and the target subsystems. In particular, it suffices to apply the adaptation of the MPS algorithm [25, 26] to SEA that was done in [24], to compute a list of dominant transmission paths in SmEdA systems. With a brief post-process, it will be also possible to automatically identify the modes more often appearing in the dominant paths. This will considerably facilitate the cumbersome task of detecting those modes regulating the energy transmission between subsystems.

The MPS algorithm in $[25,26]$ was designed to solve the so called K-shortest loopless path problem in a graph. To do so, it resorts to the Bellman's optimality principle which states that there is a shortest path 


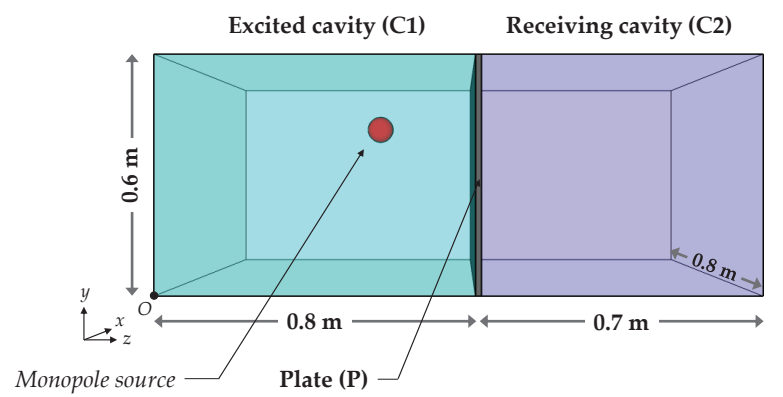

Figure 3: Cavity-panel-cavity system.

in a graph formed by shortest sub-paths. Once the shortest path between the source and target nodes has been computed, one can obtain the second-shortest path in the graph, the third-shortest path, the fourth and so on, from the analysis of their corresponding deviation paths [42]. The MPS algorithm was modified in [24] to allow one to compute maximum energy transmission paths instead of minimum ones, as well as to deal with the presence of loops.

\section{Application to cavity-panel-cavity models}

In this section, the above developments will be applied to cavity-panel-cavity models. Most conclusions in [22] with regard to modal energy transmission between cavities will be validated, but now following a totally different and faster approach. The models under analysis consist of two cavities separated apart by a panel, as shown in Fig. 3. It will be assumed that energy transmission only takes place through the dividing panel, the remaining cavity walls being totally rigid. In a first example a homogeneous panel will be considered. This comprises of a steel plate with dimensions $0.8 \mathrm{~m} \times 0.6 \mathrm{~m} \times 0.001 \mathrm{~m}$, mass density $\rho=7800 \mathrm{~kg} / \mathrm{m}^{3}$, Young modulus $\mathrm{E}=2 \times 10^{11} \mathrm{~Pa}$ and damping loss factor $\eta=0.01$. The panel is supposed to be simply-supported on its four edges. In the second example, the steel plate will be stiffened with the addition of some ribs, as detailed in Section 4.2.

The dimensions of the source cavity $\mathrm{C} 1$, are $0.8 \mathrm{~m} \times 0.6 \mathrm{~m} \times 0.8 \mathrm{~m}$, and those of the receiver cavity $\mathrm{C} 2,0.8 \mathrm{~m} \times 0.6 \mathrm{~m} \times 0.7 \mathrm{~m}$. The cavities are filled with air (mass density $\rho_{0}=1.29 \mathrm{~kg} / \mathrm{m}^{3}$, speed of sound $c_{0}=340 \mathrm{~m} / \mathrm{s}$ and damping loss factors $\eta_{C 1}=\eta_{C 2}=0.01$ ). $C 1$ will be excited with a monopole source of unit strength located at the point $(0.24,0.42,0.54) \mathrm{m}$, according to the coordinate system $[O ; x, y, z]$ in Fig. 3 . In forthcoming explanations, the particular $i$-th mode belonging to the source cavity $\mathrm{C} 1$ will be referred to as $p_{i} \in \hat{P}$, the $i$-th mode belonging to the plate as $q_{i} \in \hat{Q}$ and the $i$-th mode in the receiver cavity $\mathrm{C} 2$ as $r_{i} \in \hat{R}$. For both examples, the most dominant modal energy transmission paths have been computed for the one third octave bands ranging from $400 \mathrm{~Hz}$ to $4000 \mathrm{~Hz}$ central frequencies.

\subsection{Cavity - bare plate - cavity}

In this case, the subsystem modal information required in SmEdA can be calculated analytically for both, the natural frequencies and the interaction modal works (i.e. integral of the product between the pressure cavity modes and the displacement panel modes). In Table 1, a first overview on how modal energy transmission takes place for every analyzed $1 / 3$ octave band is presented (first column in the table). The results are built from the outputs of the the MPS algorithm as implemented in [24]. The second column in the table contains the overall energy at the receiver cavity $\mathrm{C} 2$. The third one indicates the total number of paths that have been computed for each band and the fourth and fifth columns show the contributions of the computed paths, respectively in percentage and decibels, to the energy at C2. Next, the type of paths that participate in the energy transmission from $\mathrm{C} 1$ to $\mathrm{C} 2$ are indicated (tri-block sixth column). The acronym PNR stands for purely non-resonant paths, herein identified with paths that only involve the bare plate non-resonant modes, whereas PR stands for purely resonant paths, i.e. paths only involving the plate 


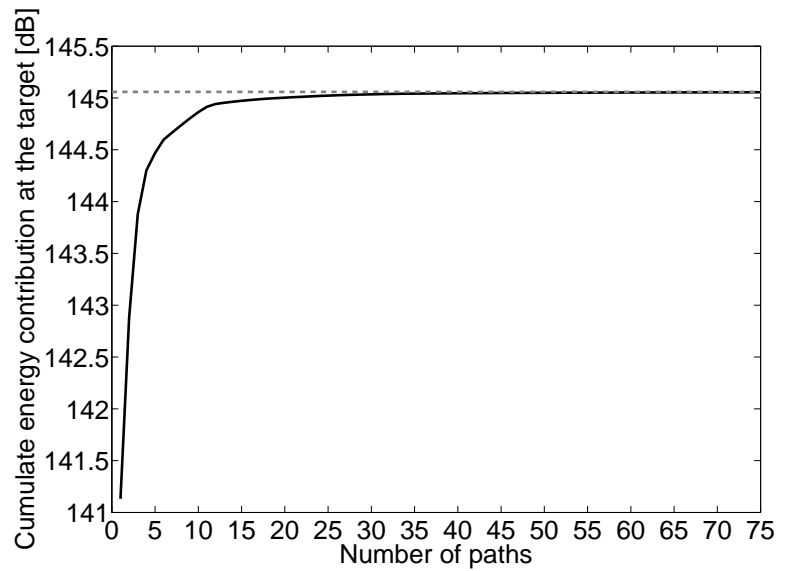

Figure 4: Cumulative path energy contribution at the receiver cavity $\mathrm{C} 2$ versus number of considered dominant paths, for the cavity - bare plate - cavity example at $630 \mathrm{~Hz}$. Continuous black line: cumulative energy contribution, dashed grey line: total energy at the receiver $\mathrm{C} 2$.

resonant modes. $\mathrm{M}$ denotes mixed transmission paths that contain both, resonant and non-resonant plate modes. The contributions in percentage and decibels of the various types of paths is exposed in the tri-block columns seven and eight.

As observed in the table, though the number of resonant paths is large and for several bands surpass that of non-resonant paths, their contribution to the energy level at C2 becomes negligible when compared to the non-resonant one, as one would expect for the behavior of a homogeneous plate well below the critical frequency $f_{c}\left(f_{c} \sim 11 \mathrm{kHz}\right.$ in the present example). Let us next focus, for the ease of exposition, on the results of the $630 \mathrm{~Hz}$ one-third octave band. There are 12 resonant modes for the source cavity $\mathrm{C} 1$ and for the receiver cavity $\mathrm{C} 2$ in this band. In what concerns the plate, it has 21 resonant modes and 75 non-resonant modes in the band. As explained, the latter do not result in extra nodes in the SmEdA graph but in additional edges directly connecting the modes of the two cavities. The SmEdA graph for the problem at hand will thus contain 47 nodes, 45 arising from all resonant modes in the system, plus two nodes corresponding to the fictitious source and target nodes (see Fig. 2).

In Fig. 4, the cumulative contribution of the first 75 paths has been plotted. The dashed line represents the total energy at the receiver cavity which has a value of $145.06 \mathrm{~dB}$ ref. $10^{-12} \mathrm{~J}$, whereas the black solid line corresponds to the cumulative energy contribution when increasing the number of considered paths. It

\begin{tabular}{|c|c|c|c|c|c|c|c|c|c|c|c|c|c|}
\hline \multirow{2}{*}{$\begin{array}{c}\text { Band } \\
{[\mathrm{Hz}]}\end{array}$} & $\begin{array}{c}\text { Cavity } \\
\text { C2 } \\
\text { Energy }\end{array}$ & $\begin{array}{c}\text { Number } \\
\text { of } \\
\text { Paths }\end{array}$ & $\begin{array}{c}\text { Accum. } \\
\text { Contr. } \\
{[\%]}\end{array}$ & $\begin{array}{l}\text { Accum. } \\
\text { Contr. } \\
{[\mathrm{dB}]}\end{array}$ & \multicolumn{3}{|c|}{ Type of paths [\%] } & \multicolumn{2}{|c|}{ Accum. Contr. [\%] } & \multicolumn{3}{|c|}{ Accum. Contr. [dB] } \\
\hline 400 & 148.06 & 100 & 100.00 & 148.06 & 16.00 & 33.00 & 51.00 & 93.96 & 5.31 & 0.73 & 147.79 & 135.32 & 126.69 \\
500 & 136.57 & 100 & 100.00 & 136.57 & 10.00 & 50.00 & 40.00 & 80.16 & 18.89 & 0.96 & 135.61 & 129.33 & 116.37 \\
630 & 145.06 & 100 & 99.95 & 145.06 & 29.00 & 69.00 & 2.00 & 95.91 & 4.02 & 0.02 & 144.88 & 131.11 & 107.39 \\
800 & 143.72 & 100 & 99.38 & 143.69 & 41.00 & 57.00 & 2.00 & 84.57 & 14.76 & 0.05 & 142.99 & 135.41 & 110.86 \\
1000 & 142.08 & 200 & 98.69 & 142.02 & 39.50 & 60.50 & 0.00 & 87.57 & 11.12 & 0.00 & 141.50 & 132.54 & $-\infty$ \\
1250 & 141.90 & 500 & 98.57 & 141.84 & 39.00 & 60.20 & 0.80 & 86.15 & 12.40 & 0.02 & 141.25 & 132.83 & 105.14 \\
1600 & 140.87 & 1000 & 97.79 & 140.77 & 39.90 & 60.10 & 0.00 & 91.41 & 6.38 & 0.00 & 140.48 & 128.92 & $-\infty$ \\
2000 & 140.87 & 1000 & 95.83 & 140.68 & 65.10 & 34.90 & 0.00 & 92.56 & 3.26 & 0.00 & 140.53 & 126.00 & $-\infty$ \\
2500 & 140.33 & 2000 & 93.72 & 140.04 & 69.75 & 30.25 & 0.00 & 90.02 & 3.69 & 0.00 & 139.87 & 126.00 & $-\infty$ \\
3150 & 140.21 & 5000 & 93.10 & 139.90 & 67.10 & 32.90 & 0.00 & 89.97 & 3.13 & 0.00 & 139.75 & 125.16 & $-\infty$ \\
4000 & 139.29 & 10000 & 91.39 & 138.90 & 71.58 & 28.42 & 0.00 & 88.76 & 2.64 & 0.00 & 138.77 & 123.50 & $-\infty$ \\
\hline
\end{tabular}

Table 1: Cavity - bare plate - cavity example. Contributions of resonant, non-resonant and mixed transmission paths for the considered $1 / 3$ octave frequency bands. 
can readily be checked, for instance, that the first 5 paths had an overall contribution of $144.47 \mathrm{~dB}$ whereas the first 75 paths supply $145.05 \mathrm{~dB}$ of energy (99.9\% of the overall energy at the target). At this point, it should be remarked that for practical applications in vibroacoustic problems differences of less than $1 \mathrm{~dB}$ become almost negligible (this constitutes in fact an eligible criteria to make a decision on the number of paths to be computed for each graph). Therefore, it can be observed that with the sole contribution of the five most dominant transmission paths it becomes possible to justify the whole transmission of energy from $\mathrm{C} 1$ to $\mathrm{C} 2$.

More insight can be gained by having a look at the results in Table 2. Its first column contains the number of transmission paths taken into account in the analysis, their cumulative energy contribution to the target subsystems in $\mathrm{dB}$ and percentage respectively being presented in columns two and three. The next three columns include the number of resonant modes of each subsystem $(\mathrm{C} 1$, panel and $\mathrm{C} 2)$ that are involved in the corresponding paths of the first column, followed by the total summation of resonant modes in column number seven. Finally, the last column of the table indicates the difference between the total number of modes in the system and the number of modes appearing in the paths of the first column.

With regard to the row information in the table, the first row is a reference containing all data involved in the exact transmission process from source to target. The second row shows the results when considering 75 dominant paths, which justify, as said, $99.9 \%$ of the energy at the receiver cavity. 38 modes from the 45 modes of the complete problem still play a role in the energy transmission process. However, by simply considering 25 paths, the accumulated contribution is $99.16 \%$, which leads to a negligible difference in terms of decibels compared to the reference (less than $0.04 \mathrm{~dB}$ ) and only 27 modes become involved in the transmission. Focusing on the 8 most dominant paths, it can be observed that the difference in dB is still insignificant (less than $1 \mathrm{~dB}$ ) and that only 14 modes are of importance. There is also a very important point to note in this case. As opposed to the preceding ones, it can be appreciated that no plate resonant mode intervenes. In other words, the energy transmission between the two cavities at the 630 one-third octave band is mainly non-resonant, as already noticed from the analysis of the results in Table 1 . The proposed graph approach not only allows one to corroborate this well-known point, but to determine which are the most important non-resonant modes dominating the transmission. In Table 3 , the ranking of the 10 stronger dominant paths has been listed. Non-resonant paths can be easily identified as they do not contain any $q_{i}$ element. As seen in the table, the first eight transmission paths are completely non-resonant, being the ninth path the first one to include a $q_{i}$ mode (in particular $q_{21}$ ).

It should be remarked that obtaining the type of information in Tables 1, 2 and 3 is a straightforward task from the output of the MPS algorithm. As opposed to this, trying to get this kind of information directly from the analysis of the whole SmEdA system is a rather intricate and lengthy task that involves several simplifying hypotheses [22]. This can be realized when trying to determine the first two or three dominant paths in the SmEdA model following the procedure in [22]. To do so, the SmEdA model has to be first simplified assuming weak coupling and taking advantage of the fact that the three subsystems are connected in series. Then, it may be expected that the modal energies of the excited subsystem $C 1$ will be much higher than those of the homogeneous plate, which in turn will be higher than those in the receiving cavity $C 2$. The simplified SmEdA model relates the modal energies of $C 2$ with the modal injected powers through non-resonant and resonant paths, by means of equations (51) and (52) in [22], respectively. Inspecting the values of the analytical factors relating these quantities, it becomes possible to determine

\begin{tabular}{|c|c|c|c|c|c|c|c|}
\hline $\begin{array}{c}\text { Number } \\
\text { of paths }\end{array}$ & $\begin{array}{c}\text { Accumulated } \\
\text { contribution } \\
{[\mathrm{dB}]}\end{array}$ & $\begin{array}{c}\text { Accumulated } \\
\text { contribution } \\
{[\%]}\end{array}$ & $\hat{P}$ & $\hat{Q}^{R}$ & $\hat{R}$ & $\begin{array}{c}\text { Total } \\
\text { involved } \\
\text { modes }\end{array}$ & $\begin{array}{c}\text { Missing } \\
\text { modes }\end{array}$ \\
\hline$\infty$ & 145.06 & 100.00 & 12 & 21 & 12 & 45 & 0 \\
75 & 145.05 & 99.91 & 12 & 14 & 12 & 38 & 7 \\
25 & 145.02 & 99.16 & 12 & 5 & 10 & 27 & 18 \\
10 & 144.86 & 95.59 & 7 & 1 & 7 & 15 & 30 \\
8 & 144.73 & 92.82 & 7 & 0 & 7 & 14 & 31 \\
\hline
\end{tabular}

Table 2: Cavity - bare plate - cavity example. Transmission path analysis and involved modes for the $630 \mathrm{~Hz} 1 / 3$ octave band. 


\begin{tabular}{|c|l|}
\hline Path & Cavity - Homogenous plate - cavity \\
\hline 1 & $S \rightarrow p_{6} \rightarrow r_{7} \rightarrow T$ \\
2 & $S \rightarrow p_{10} \rightarrow r_{10} \rightarrow T$ \\
3 & $S \rightarrow p_{12} \rightarrow r_{11} \rightarrow T$ \\
4 & $S \rightarrow p_{1} \rightarrow r_{3} \rightarrow T$ \\
5 & $S \rightarrow p_{4} \rightarrow r_{5} \rightarrow T$ \\
6 & $S \rightarrow p_{3} \rightarrow r_{6} \rightarrow T$ \\
7 & $S \rightarrow p_{6} \rightarrow r_{10} \rightarrow T$ \\
8 & $S \rightarrow p_{7} \rightarrow r_{9} \rightarrow T$ \\
9 & $S \rightarrow p_{12} \rightarrow q_{21} \rightarrow r_{11} \rightarrow T$ \\
10 & $S \rightarrow p_{10} \rightarrow r_{7} \rightarrow T$ \\
\hline
\end{tabular}

Table 3: Cavity - bare plate - cavity example. Ranking of the 10 most dominant modal energy paths from a total of 75 computed paths for the $630 \mathrm{~Hz} 1 / 3$ octave band

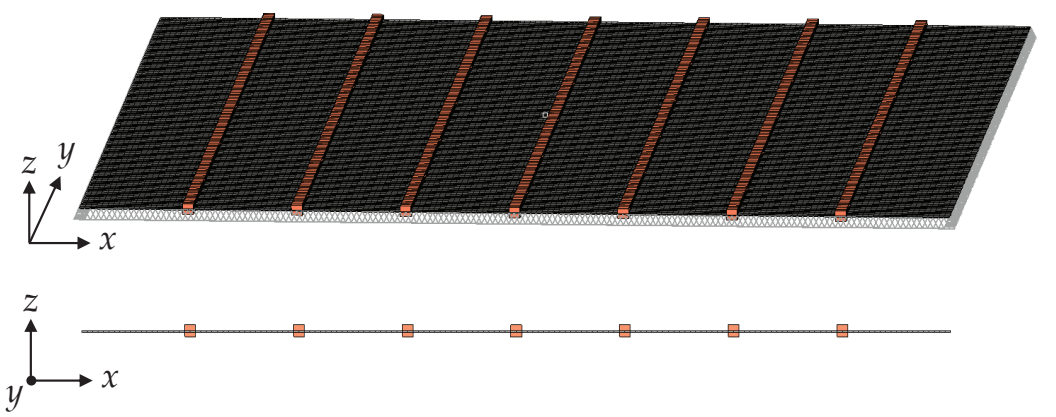

Figure 5: Ribbed plate finite element model

the two or three most dominant paths, which coincide with those in the ranking of Table 3 . Therefore, one can resort to the procedure in [22] to validate the first paths resulting from the application of the MPS algorithm, but it should be emphasized that by no means is it possible to apply that procedure to generate an automatic list of dominant paths. Moreover, the approach in [22] only works under some restrictive assumptions which may not be valid in the case of complex SmEdA models. As seen, such difficulties can be overcome by resorting to the MPS algorithm.

\subsection{Cavity - ribbed plate - cavity}

In this second example the homogeneous bare plate becomes stiffened with some ribs. The ribs are regularly placed $100 \mathrm{~mm}$ apart parallel to the plate $y$-axis and have a cross section of $10 \mathrm{~mm} \times 10 \mathrm{~mm}$, see Fig. 5. It is well-known that the acoustic transmission of a ribbed plate differs from that of a bare plate because its stiffness becomes increased in the rib direction. As a first approximation for low frequencies, the ribbed plate may be considered equivalent to an orthotropic plate. Whereas the bare plate has a single critical frequency ( $\sim 11 \mathrm{kHz}$ in the previous example), an orthotropic plate is characterized by having two critical frequencies. The lowest one is that corresponding to the coincidence of waves traveling in the plate stiffest direction (rib direction) with acoustic waves. In our case, this first critical frequency occurs at $\sim 1.7 \mathrm{kHz}$ (the second being that of the bare plate at $\sim 11 \mathrm{kHz}$ ). Therefore and contrary to the bare plate, for which resonant plate modes are only in spatial coincidence with the acoustic modes beyond $11 \mathrm{kHz}$, the stiffened plate may have some resonant modes in spatial coincidence with the acoustic ones for frequencies well below $11 \mathrm{kHz}$. One would then expect the resonant paths to play a more important role in the acoustic transmission than the one they played for the bare plate. It is however difficult to make an a priori estimation of the importance of the resonant paths compared to the non-resonant ones. As it will be shown below the graph theory approach can substantially ease this task.

In order to build the SmEdA matrix for this case, the subsystem modes of the ribbed plate have been calculated with FEM. The plate has been modelled with 19200 quadrilateral shell elements while 900 onedimensional beam elements have been used for the ribs (see Fig. 5). Uncoupled extensional (in-plane) 


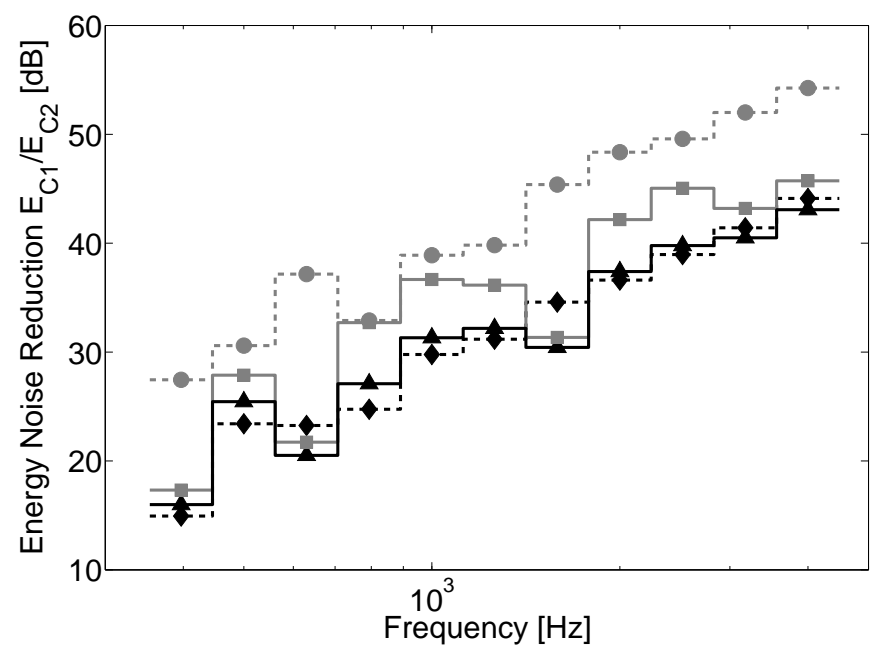

Figure 6: Energy ratio between the two cavities. - - - - (grey) bare plate with only resonant transmission, - - - - (black) bare plate with resonant and non-resonant transmission, _- - (grey) ribbed plate with only resonant transmission — - - (black) ribbed plate with resonant and non-resonant transmission.

and bending (out-plane) motions have been considered for the thin plate whereas bending, extensional and torsional motions have been taken into account for the beam-like stiffeners. Based on the classical FEM sampling criterion of using six elements per wavelength in the generated mesh, the results are expected to be valid up to $10 \mathrm{kHz}$. The normal modes have been computed using the SDTools code [43]. Besides, the cavity modes have been calculated analytically as in the cavity-bare plate-cavity example of the preceding subsection.

As expected, the energy transmission no longer presents two distinct ranges of behavior like for the bare plate. This can be first observed in Table 4, built again from the outputs of the MPS algorithm, which is the analogous to Table 1 but for the ribbed case. As seen, it is no longer true that the contribution to the energy level at $\mathrm{C} 2$ becomes dominated by non-resonant paths, the resonant ones playing a determinant role for several $1 / 3$ octave bands. More graphically, this can be appreciated when analyzing the energy noise reduction (ENR) between cavities [22]. The ENR is defined as $E N R=10 \log _{10}\left(E_{C 1} / E_{C 2}\right)$, with $E_{C 1}$ and $E_{C 2}$ respectively standing for the time averaged total energies at cavities $\mathrm{C} 1$ and $\mathrm{C} 2$ respectively. In Fig. 6 the ENR has been plotted in one-third octave bands for both, the bare plate of the previous example

\begin{tabular}{|c|c|c|c|c|c|c|c|c|c|c|c|c|c|}
\hline \multirow{2}{*}{$\begin{array}{c}\text { Band } \\
{[\mathrm{Hz}]}\end{array}$} & \multirow{2}{*}{$\begin{array}{l}\text { Target } \\
\text { Energy }\end{array}$} & \multirow{2}{*}{$\begin{array}{c}\text { Number } \\
\text { of } \\
\text { Paths }\end{array}$} & \multirow{2}{*}{$\begin{array}{c}\text { Accum. } \\
\text { Contr. } \\
{[\%]}\end{array}$} & \multirow{2}{*}{$\begin{array}{c}\text { Accum. } \\
\text { Contr. } \\
{[\mathrm{dB}]}\end{array}$} & \multicolumn{3}{|c|}{ Type of paths [\%] } & \multicolumn{3}{|c|}{ Accum. Contr. [\%] } & \multicolumn{3}{|c|}{ Accum. Contr. [dB] } \\
\hline & & & & & PNR & $\mathrm{PR}$ & M & PNR & $\mathrm{PR}$ & M & PNR & $\mathrm{PR}$ & M \\
\hline 400 & 146.84 & 100 & 99.84 & 146.83 & 7.00 & 68.00 & 25.00 & 25.83 & 73.28 & 0.73 & 140.96 & 145.49 & 125.45 \\
\hline 500 & 133.89 & 100 & 99.93 & 133.89 & 6.00 & 75.00 & 19.00 & 42.60 & 56.89 & 0.44 & 130.19 & 131.44 & 110.37 \\
\hline 630 & 147.46 & 200 & 99.65 & 147.44 & 11.50 & 83.50 & 5.00 & 24.36 & 75.24 & 0.05 & 141.33 & 146.22 & 114.63 \\
\hline 800 & 141.37 & 200 & 99.49 & 141.36 & 23.00 & 75.50 & 1.50 & 72.43 & 27.04 & 0.03 & 139.97 & 135.69 & 105.93 \\
\hline 1000 & 140.53 & 300 & 99.48 & 140.51 & 27.00 & 72.00 & 1.00 & 70.77 & 28.69 & 0.02 & 139.03 & 135.11 & 104.14 \\
\hline 1250 & 140.88 & 1000 & 98.79 & 140.83 & 17.70 & 82.10 & 0.20 & 59.76 & 39.03 & 0.01 & 138.64 & 136.79 & 98.38 \\
\hline 1600 & 144.98 & 2000 & 98.55 & 144.91 & 16.00 & 83.55 & 0.45 & 18.92 & 79.62 & 0.02 & 137.74 & 143.99 & 107.25 \\
\hline 2000 & 140.07 & 2000 & 94.89 & 139.84 & 33.35 & 66.65 & 0.00 & 66.07 & 28.82 & 0.00 & 138.27 & 134.67 & $-\infty$ \\
\hline 2500 & 139.50 & 5000 & 92.79 & 139.17 & 35.08 & 64.92 & 0.00 & 69.51 & 23.29 & 0.00 & 137.92 & 133.17 & $-\infty$ \\
\hline 3150 & 141.12 & 10000 & 82.84 & 140.30 & 27.56 & 72.44 & 0.00 & 44.81 & 38.03 & 0.00 & 137.63 & 136.92 & $-\infty$ \\
\hline 4000 & 140.32 & 10000 & 78.70 & 139.28 & 42.98 & 57.02 & 0.00 & 42.66 & 36.05 & 0.00 & 136.62 & 135.89 & $-\infty$ \\
\hline
\end{tabular}

Table 4: Cavity - ribbed plate - cavity example. Contributions of resonant, non-resonant and mixed transmission paths for the considered $1 / 3$ octave frequency bands. 


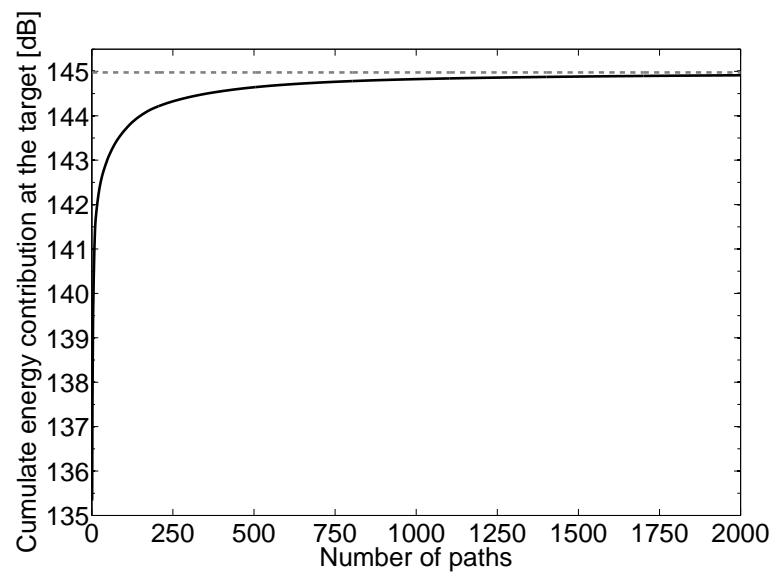

Figure 7: Cumulative path energy contribution at the receiver cavity $\mathrm{C} 2$ versus number of considered dominant paths, for the cavity - ribbed plate - cavity example for the $1600 \mathrm{~Hz} 1 / 3$ octave band. Continuous black line: cumulative energy contribution, Continuous straight grey line: total energy at the receiver C2.

(dashed lines) and the ribbed one (solid lines). For each case two ENRs have been considered, the first one only considers resonant modes in the transmission (grey lines) whereas the second one takes into account resonant and non-resonant transmission as well (black lines).

Given that the maximum frequency limit in the figure is $4 \mathrm{KHz}$, which is well below the critical frequency of the bare plate, the resonant transmission ENR (dashed grey line) is clearer higher than the resonant plus non-resonant ENR (dashed black line) because, as explained in Section 4.1 (see Table 1), transmission is mainly dominated by non-resonant modes at this frequency range. However, when comparing the ENRs for the ribbed plate, there is no clear dominance from one type of transmission or another. Besides, note from Fig. 6 that the ENR of the ribbed plate considering all type of paths is generally higher than that of the bare plate, except for the $630 \mathrm{~Hz}$ and $1600 \mathrm{~Hz}$ bands. At these frequencies, the transmission seems to be mainly resonant for the ribbed plate, since the grey and black solid lines have really close values and there are two dips in the ENR curves. This is confirmed by the results in Table 4.

If the transmission is mostly resonant, one could suspect that there is a group of modes of the cavities and the plate which exhibit spatial and frequency coincidences, i.e. a group involving a path of the type $p_{i} \rightarrow q_{i} \rightarrow r_{i}$. One could then be tempted to check from all possible combinations of three modes which of them present a high intermodal work $W_{i j}$ (to account for the spatial matching) or which are closer in frequency (to account for the frequency matching), to try to determine which modes control the energy coupling. However, this is not a very good option because the effects of spatial and frequency coincidence are studied separately. To determine modal energy transmission it is better to focus the analysis on the modal coupling factors, see (5), which include the spatial and frequency coincidence effects at the same time. Inspecting the values of these factors between the excited cavity and the ribbed panel on the one side, and between the ribbed panel and the receiving cavity on the other side, it is possible to identify the most dominant paths between each pair of coupled subsystems. However, it still becomes very difficult to identify the most dominant paths when considering the coupling of all three subsystems together. For example, one plate mode could be strongly coupled with a mode of the excited cavity whereas poorly coupled with the modes of the receiving cavity. Such a mode could exchange much less energy than another one being moderately coupled with the modes of both cavities. The analysis of the modal coupling may be a lengthy and time consuming manual procedure which cannot fully guarantee that the inspected modes will be the ones dominating energy transmission. Alternatively, resorting to graph theory allows one to gather this type of information.

It is interesting to focus on the $1600 \mathrm{~Hz}$ frequency band for which resonant modes play a significant role. In Fig. 7 the cumulative energy contribution of the first 2000 paths in this band is depicted. The total energy at the receiver cavity is $144.97 \mathrm{~dB}$ and the contribution e.g., of the first 250 paths already provides $144.32 \mathrm{~dB}$, 


\begin{tabular}{|c|l|}
\hline Path & Cavity - Ribbed plate - cavity \\
\hline 1 & $S \rightarrow p_{49} \rightarrow q_{20} \rightarrow r_{49} \rightarrow T$ \\
2 & $S \rightarrow p_{7} \rightarrow q_{1} \rightarrow r_{13} \rightarrow T$ \\
3 & $S \rightarrow p_{14} \rightarrow q_{5} \rightarrow r_{12} \rightarrow T$ \\
4 & $S \rightarrow p_{82} \rightarrow q_{31} \rightarrow r_{72} \rightarrow T$ \\
5 & $S \rightarrow p_{82} \rightarrow q_{31} \rightarrow r_{55} \rightarrow T$ \\
6 & $S \rightarrow p_{78} \rightarrow q_{20} \rightarrow r_{49} \rightarrow T$ \\
7 & $S \rightarrow p_{49} \rightarrow q_{20} \rightarrow r_{30} \rightarrow T$ \\
8 & $S \rightarrow p_{141} \rightarrow q_{57} \rightarrow r_{129} \rightarrow T$ \\
9 & $S \rightarrow p_{82} \rightarrow q_{31} \rightarrow r_{81} \rightarrow T$ \\
10 & $S \rightarrow p_{33} \rightarrow q_{20} \rightarrow r_{49} \rightarrow T$ \\
\hline
\end{tabular}

Table 5: Cavity - ribbed plate - cavity example. Ranking of the 10 most dominant modal energy paths from a total of 2000 computed paths for the $1600 \mathrm{~Hz} 1 / 3$ octave band.

which is fairly close to the total value (less than $1 \mathrm{~dB}$ ). Therefore, it becomes feasible to circumscribe the analysis to the ranking of the first 250 paths. For every mode, the number of appearances in the list of the 250 paths and the number of paths that contain it have been counted. The results are presented in Fig. 8. It can be observed that for every subsystem, there is a relatively small group of outstanding modes, whose maxima can respectively be identified as $p_{49}, q_{20}$ and $r_{49}$. One may presume that these modes will play a predominant role in energy transmission. Actually, if one inspects the ranking of the 250 paths (see Table 5 for the first 10 paths), it precisely follows that the preeminent path is $p_{49} \rightarrow q_{20} \rightarrow r_{49}$ which contributes

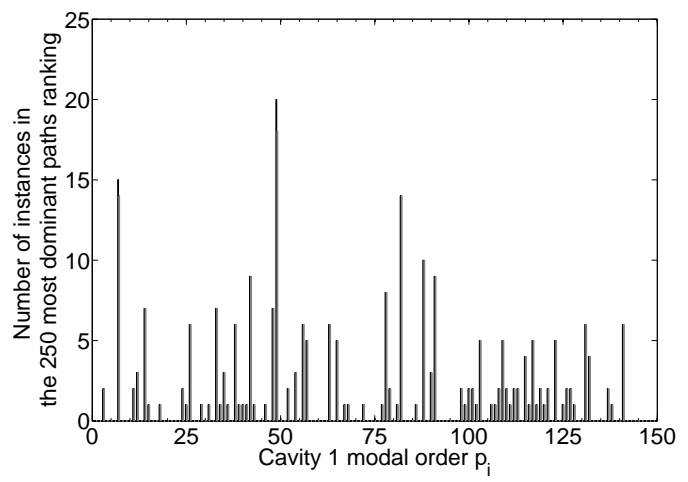

(a) Cavity 1

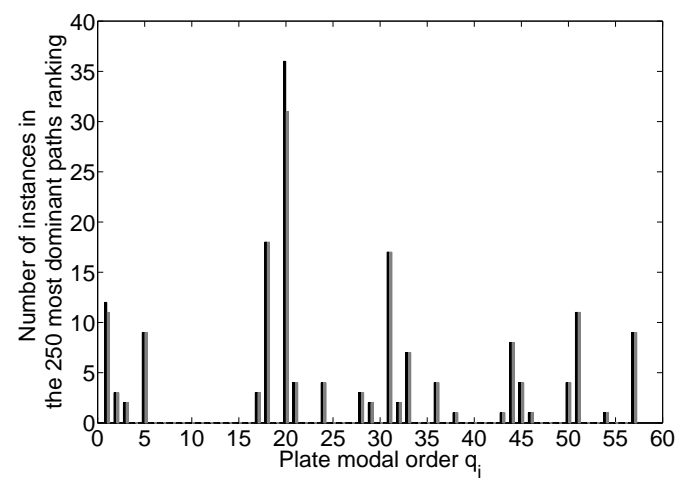

(b) Plate

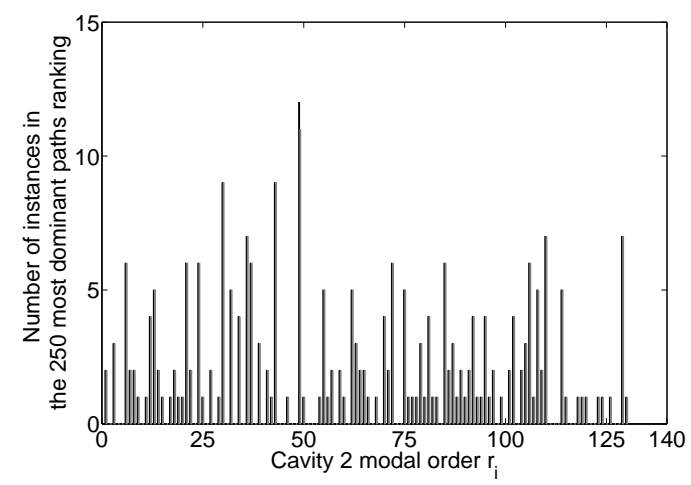

(c) Cavity 2

Figure 8: Number of instances of every mode in the 250 most dominant paths ranking. Black columns: total number of instances in the path ranking. Grey columns: number of paths containing a particular mode. 
$135.36 \mathrm{~dB}$ to the energy at the receiver cavity. The natural frequencies of these modes are $1547 \mathrm{~Hz}$ for $p_{49}$, $1549 \mathrm{~Hz}$ for $q_{20}$, and $1565 \mathrm{~Hz}$ for $r_{49}$. These modes are relatively close in frequency but for the receiving cavity, other modes closer to $q_{20}$ than $r_{49}$ can be found. For example, $r_{42}$ has a frequency of $1547 \mathrm{~Hz}$. Thus, the matching in frequency cannot explain in itself why $p_{49} \rightarrow q_{20} \rightarrow r_{49}$ dominates.

It is worth examining this point in more detail. In Fig. 9 the spatial distribution (pressure for cavity modes and displacement for the ribbed plate) on the plate surface is plotted, for the three involved modes. Although the natural flexural wavelength of the plate is $\sim 0.08 \mathrm{~m}$ at $1.5 \mathrm{kHz}$, a wavelength for mode $q_{20}$ along the $y$-direction can be appreciated well above this value, which is comparable to the wavelengths of acoustic modes. This is due to the stiffness effect of the ribs and it leads to a spatial matching between this plate mode and the acoustic modes $p_{49}$ and $r_{49}$ in the the $y$-direction. The intermodal works between $p_{49}$ and $q_{20}$, and $q_{20}$ and $r_{49}$ are, respectively, 66.7 J and $71.4 \mathrm{~J}$ and their modal coupling factors turn out to be $22.5 \mathrm{~Hz}$ and $12.8 \mathrm{~Hz}$. The latter is lower than the former whereas the opposite happens for the intermodal works. This can be simply explained by the fact that $p_{49}$ and $q_{20}$ are closer in frequency than $q_{20}$ and $r_{49}$. The spatial matching is here the key phenomenon which leads the path $p_{49} \rightarrow q_{20} \rightarrow r_{49}$ to be the most dominant one, as identified by the MPS algorithm.

In order to give the reader a point for comparison, an analysis is made of the interactions in the path $p_{34} \rightarrow q_{14} \rightarrow r_{33}$ which does not appear in the MPS ranking of the first 250 paths, though its modes have very close natural frequencies $(1514.2 \mathrm{~Hz}, 1512.0 \mathrm{~Hz}, 1512.6 \mathrm{~Hz}$, respectively). The pressure and displacement spatial patterns of those modes on the plate surface have been plotted in Fig. 10 to be compared with those in Fig. 9. As observed, the plate mode $q_{14}$ presents short wavelengths along the $x$ and $y$ directions

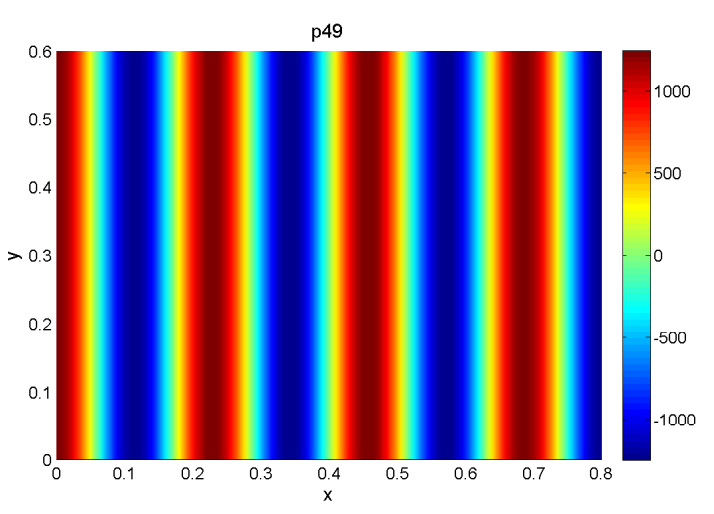

(a)

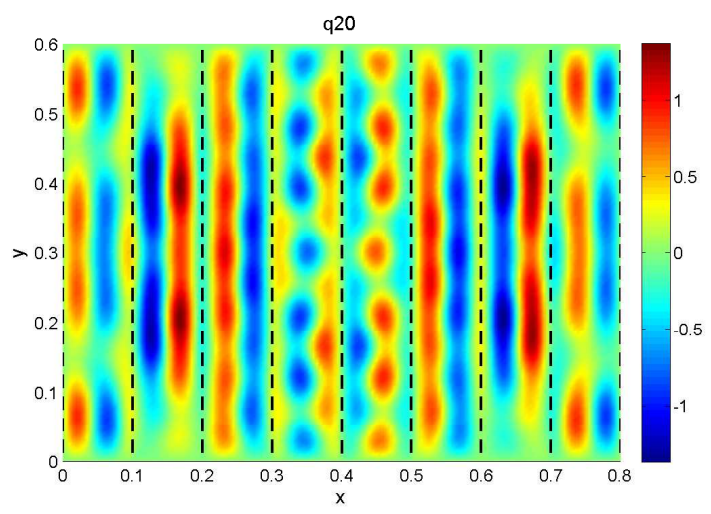

(b)

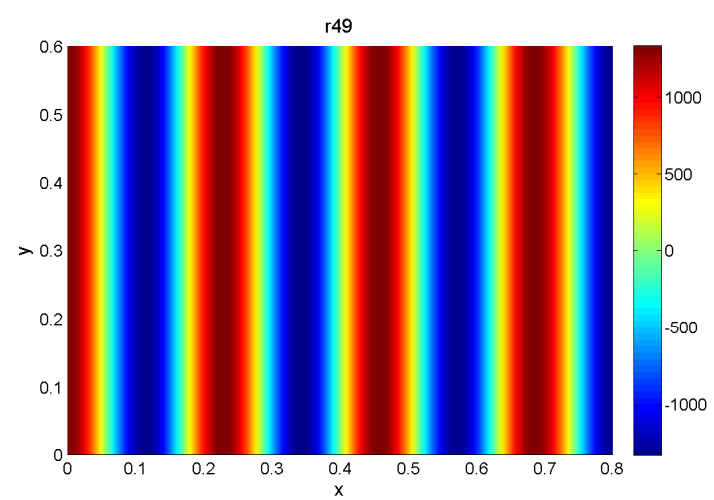

(c)

Figure 9: Magnitudes of the mode spatial shapes on the coupling surface: (a) p49; (b) q20 (Vertical dashed line: rib positions); (c) $\mathrm{r} 49$. 


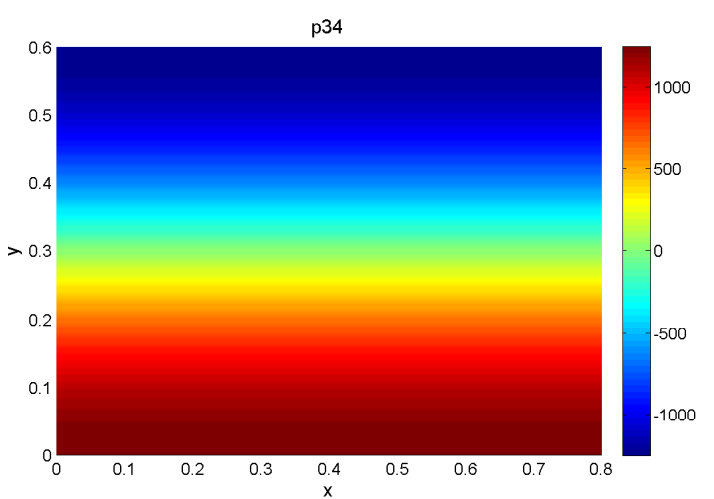

(a)

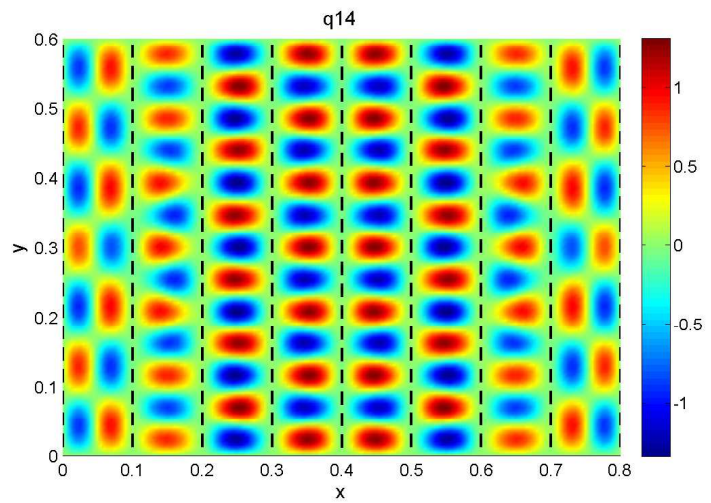

(b)

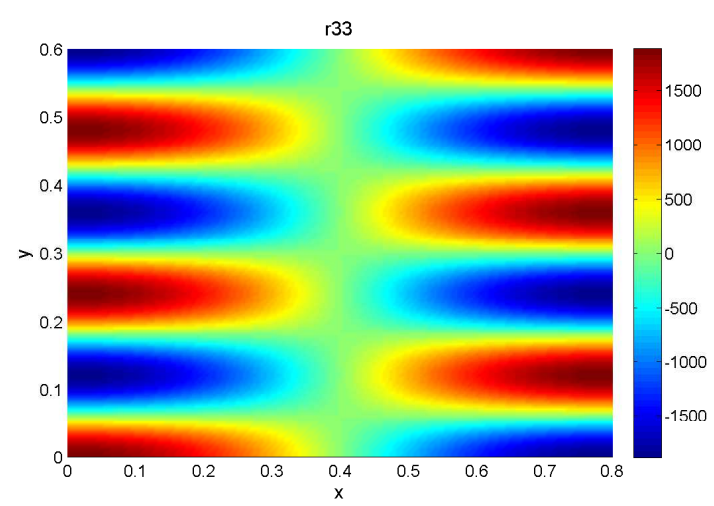

(c)

Figure 10: Magnitudes of the mode spatial shapes on the coupling surface: (a) p34; (b) q14 (Vertical dashed lines: rib positions); (c) r33.

compared to the wavelengths of the acoustic modes. This results in low values for the intermodal works $\left(1.9 \times 10^{-7} \mathrm{~J}\right.$ between $p_{34}$ and $q_{14}$ and $8.7 \times 10^{-7} \mathrm{~J}$ between $q_{14}$ and $\left.r_{33}\right)$ and in very low values for the modal coupling factors $\left(1.9 \times 10^{-16} \mathrm{~Hz}\right.$ between $p_{34}$ and $q_{14}$ and $3.9 \times 10^{-17} \mathrm{~Hz}$ between $q_{14}$ and $\left.r_{33}\right)$, despite the frequency matching. Hence, it becomes clear that the main difference between the paths $p_{49} \rightarrow q_{20} \rightarrow r_{49}$ and $p_{34} \rightarrow q_{14} \rightarrow r_{33}$ is the effect of spatial matching. The former path is the most dominant one and this is a consequence of the rib effects which lead to a low energy noise reduction in comparison to that of the bare plate for the $1600 \mathrm{~Hz}$ third octave band (see Fig. 6). Therefore, it becomes clear again how from an automatic and quick inspection of the outputs of the MPS algorithm it is possible to identify those modes responsible of a sudden decrease in the ENR curves, and act upon them if necessary. Otherwise, one could waste lots of time analysing paths with good frequency matching but no relevant energy contribution such as $p_{34} \rightarrow q_{14} \rightarrow r_{33}$.

\section{Application to a shipbuilding structure}

\subsection{Path analysis in $S m E d A$}

The above developments will be applied in this section to a more complex case consisting of a shipbuilding structure made of 6 rooms distributed in two decks (see Fig. 11). The floor between decks and the vertical walls between rooms are made of steel $\left(\rho=7800 \mathrm{~kg} / \mathrm{m}^{3}\right.$, Young modulus $E=2 \times 10^{11} \mathrm{~Pa}$ and internal damping $\eta=0.01$ ). The thicknesses of the panels are $6 \mathrm{~mm}$ for the floor and $2 \mathrm{~mm}$ for the walls. The floor and the walls are stiffened with 6 T-shaped ribs. These ribs are regularly spaced along the floor's longest 


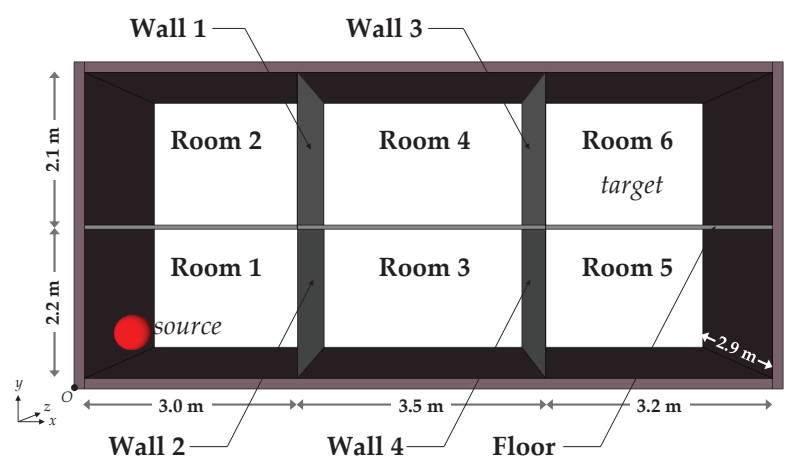

Figure 11: Sketch of the shipbuilding built-up structure model

edge and along the vertical wall edges. The rib spacing is $0.4 \mathrm{~m}$ and the first and the last ribs are respectively placed at $0.46 \mathrm{~m}$ and $0.44 \mathrm{~m}$ from the edges. The T-cross section dimensions are $[80 \times 8] \mathrm{mm}^{2} /[80 \times 8] \mathrm{mm}^{2}$ for the floor ribs and $[60 \times 5] \mathrm{mm}^{2} /[60 \times 5] \mathrm{mm}^{2}$ for the wall ones. Besides, to deal with a more amenable model the external walls and floors of the structure have been assumed rigid. The rooms are filled with air (mass density $\rho_{0}=1.29 \mathrm{~kg} / \mathrm{m}^{3}$, wavespeed $c_{0}=340 \mathrm{~m} / \mathrm{s}$, damping loss factors $\eta=0.01$ ). Overall, this results in a model with eleven subsystems: six rooms $R 1-R 6$, the floor $F$ and four separating walls $W 1-W 4$ (see Fig. 11).

In this case, to build the SmEdA model only flexural vibration modes have been considered for the panels. Different studies on ship structures $[44,45]$ showed that this type of vibration transmission is predominant in the low and mid frequency range. For high frequencies (several $\mathrm{kHz}$ for typical naval frames), in-plane modes may be of importance for some one third octave bands. Actually, it has been noticed that in-plane longitudinal and shear motions may have significant contributions on the energy transmitted to a subsystem far away from the excited one [46, 47]. However, given that the frequency range of analysis has been fixed to $[80 \mathrm{~Hz}-500 \mathrm{~Hz}]$, which is below the first in-plane mode of the floor $(616 \mathrm{~Hz})$, contemplating only bending motions is a fair enough approximation. The modes of the floor and walls have been computed with FEM and the SDTool code [43], whereas the cavity modes have been calculated analytically, like in the previous examples.

An acoustic monopole has been located at point $M_{0}=(0.8,0.9,0.7) \mathrm{m}$ in Room 1 (source subsystem), with unit power spectral density. Room 6 is considered as the receiver. In Fig. 13(a) the ENR between $\mathrm{R} 1$ and R6 has been plotted, whereas the ENR for the two adjacent rooms R1 and R3 is also presented in Fig. 13(b) for comparison. As observed, the energy reduction between R1 and R6 is considerably high except for the $200 \mathrm{~Hz}$ band where strong energy transmission takes place. One may then wonder which modes are responsible for the transmission at this frequency band to act upon them e.g., by increasing their damping and thus reducing the energy level at the receiver.

In Table 6 a path analysis is presented for the shipbuilding built-up structure, analogous to that in

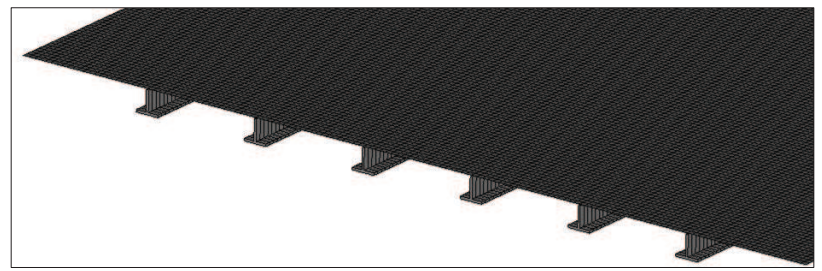

(a)

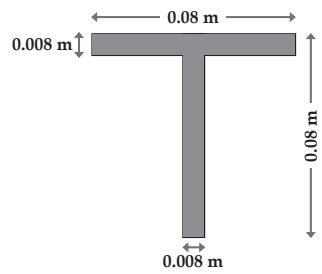

(b)

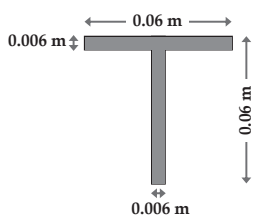

(c)

Figure 12: (a) Zoom view of the floor in the shipbuilding structure of Fig. 11. (b) Section of the floor ribs. (c) Section of the wall ribs. 


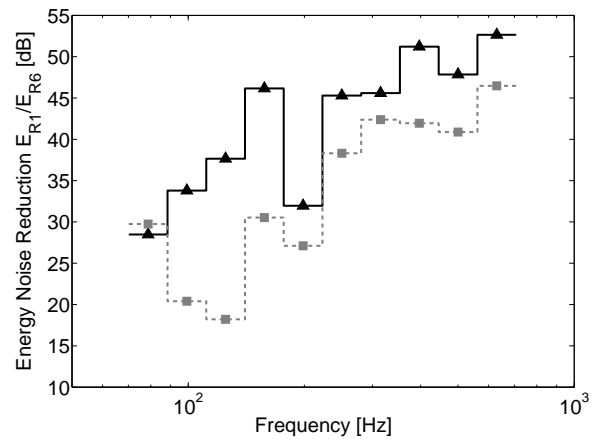

(a)

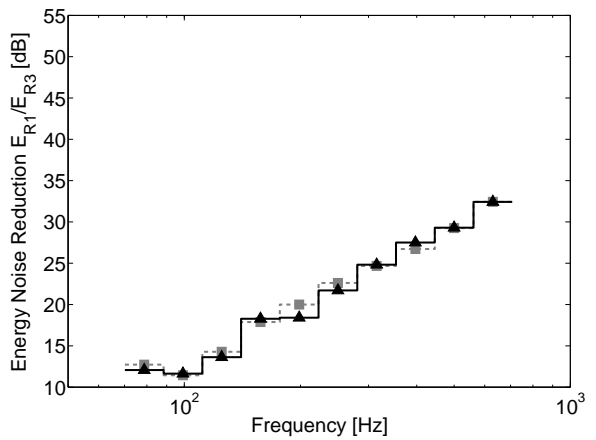

(b)

Figure 13: (a) Noise Energy Reduction $E_{R 6} / E_{R 1}$ between Room 1 and Room 6. — - - (black) SmEdA results. - - $\mathbf{-}-$ - (grey) SEA results. (b) Noise Energy Reduction $E_{R 3} / E_{R 1}$ between Room 1 and Room 3. - - - (black) SmEdA results. - - $\mathbf{-}$ - (grey) SEA results.

Table 2 for the cavity-homogeneous plate-cavity system. As seen from the table, there are a total of 326 modes involved in the $200 \mathrm{~Hz}$ band, the overall energy at the receiver subsystem being $45.06 \mathrm{~dB}$ (first row). Considering 1000 paths $93.77 \%$ of the energy at the receiver can be recovered, with a drastic reduction in the number of involved modes (from 326 to 112). The strongest reduction of modes takes place for subsystems $R 2, R 3$ and $W 1-W 4$, which is logical. With the sole consideration of 25 paths the reconstructed energy at the receiver is less than one $\mathrm{dB}$ of its overall value, which may suffice for the analysis of the vibroacoustic behaviour of the structure.

Computing the number of instances of every mode in the ranking of paths (see Fig. 14) reveals that the dominant modes of the floor are $f_{22}$ and $f_{23}$. This can also be appreciated in the ranking list of Table 7 for the first seven dominating transmission paths. These paths contribute $58.11 \%$ of the energy at the receiver and only contain modes belonging to the source room, the receiver room and the floor. This indicates that energy is mainly transmitted along the floor separating the two decks.

Similarly to what occurred for the cavity - ribbed plate - cavity example, a close inspection of modes $f_{22}$ and $f_{23}$ shows that they present high wavelengths in the floor's stiffest direction, which results in good spatial matching with the cavity modes (see Figs. 15a, b). Despite the amplitudes of the mode shapes being smaller in the junction area with room 1 than with room 6 , there is still good spatial matching of $f_{22}$ and $f_{23}$ with the cavity modes of the former. In contrast, mode $f_{25}$, for example, exhibits lower vibration values in the junction areas with rooms 1 and 6 than in the junction with room 2 , and also presents shorter wavelengths than $f_{22}$ and $f_{23}$ (see Fig. 15c). This explains the lack of spatial matching leading to destructive interference between the floor mode and the cavity modes. In turn, that results in low intermodal works within room 1 and room 6 modes.

As a consequence, one could attempt to increase the damping of modes $f_{22}$ and $f_{23}$ in order to diminish the energy level in the receiver room. This has been simulated by setting their new internal damping values

\begin{tabular}{|c|c|c|c|c|c|c|c|c|c|c|c|c|c|c|c|}
\hline $\begin{array}{l}\text { Number } \\
\text { of paths }\end{array}$ & $\begin{array}{c}\text { Accum. } \\
\text { Contr. } \\
{[\mathrm{dB}]}\end{array}$ & $\begin{array}{c}\text { Accum. } \\
\text { Contr. } \\
{[\%]}\end{array}$ & $\hat{R} 1$ & $\hat{R} 2$ & $\hat{R 3}$ & $\hat{R 4}$ & $\hat{R 5}$ & $\hat{R} 6$ & $\hat{F}$ & $\hat{W} 1$ & $\hat{W} 2$ & $\hat{W} 3$ & $\hat{W} 4$ & $\begin{array}{c}\text { Total } \\
\text { involved } \\
\text { modes }\end{array}$ & $\begin{array}{c}\text { Missing } \\
\text { modes }\end{array}$ \\
\hline$\infty$ & 45.06 & 100.00 & 15 & 14 & 18 & 18 & 17 & 17 & 65 & 42 & 40 & 40 & 40 & 326 & 0 \\
\hline 1000 & 44.78 & 93.77 & 12 & 5 & 6 & 13 & 8 & 17 & 37 & 2 & 5 & 7 & 0 & 112 & 214 \\
\hline 500 & 44.67 & 91.39 & 11 & 5 & 3 & 8 & 4 & 15 & 30 & 1 & 3 & 5 & 0 & 85 & 241 \\
\hline 100 & 44.44 & 81.67 & 8 & 3 & 3 & 4 & 3 & 12 & 14 & 0 & 0 & 2 & 0 & 49 & 277 \\
\hline 25 & 44.18 & 70.85 & 3 & 1 & 0 & 0 & 1 & 5 & 6 & 0 & 0 & 0 & 0 & 16 & 310 \\
\hline 7 & 42.70 & 58.11 & 1 & 0 & 0 & 0 & 0 & 3 & 3 & 0 & 0 & 0 & 0 & 7 & 319 \\
\hline
\end{tabular}

Table 6: Shipbuilding structure. Transmission path analysis and involved modes. 


\begin{tabular}{|c|l|}
\hline Path & \multicolumn{1}{|c|}{ Shipbuilding } \\
\hline 1 & $S \rightarrow r 1_{6} \rightarrow f_{22} \rightarrow r 6_{5} \rightarrow T$ \\
2 & $S \rightarrow r 1_{6} \rightarrow f_{22} \rightarrow r 6_{6} \rightarrow T$ \\
3 & $S \rightarrow r 1_{6} \rightarrow f_{23} \rightarrow r 6_{5} \rightarrow T$ \\
4 & $S \rightarrow r 1_{6} \rightarrow f_{23} \rightarrow r 6_{6} \rightarrow T$ \\
5 & $S \rightarrow r 1_{6} \rightarrow f_{22} \rightarrow r 6_{7} \rightarrow T$ \\
6 & $S \rightarrow r 1_{6} \rightarrow f_{21} \rightarrow r 6_{5} \rightarrow T$ \\
7 & $S \rightarrow r 1_{6} \rightarrow f_{23} \rightarrow r 1_{6} \rightarrow f_{22} \rightarrow r 6_{5} \rightarrow T$ \\
\hline
\end{tabular}

Table 7: Shipbuilding structure example. Ranking of the 10 most dominant modal energy paths from a total of 1000 computed paths

to $\eta_{22}=\eta_{23}=0.1$, which has resulted in a $5.0 \mathrm{~dB}$ reduction of the transmitted energy. Of course this structural modification is only theoretical, but it confirms that the structural modes on which one should intervene for controlling the noise transmission have been well identified. In practice, viscoelastic layers can be used for increasing the modal damping. In order to optimize the increase of damping of a given mode the layers should be placed at the positions of maximum strain energy according to the mode shape [48]. The damping factors of all modes in the considered structure will be obviously influenced by these viscoelastic layers. They could be estimated using the Modal Strain Energy (MSE) method [49-51].

To summarize, the MPS algorithm has allowed for a quick identification of the two most critical modes determining the energy transmission between source and receiver in the SmEdA model of the shipbuilding built-up structure, which contains a total of 326 modes. The sole modification of the damping loss factors of two modes, from 65 modes in the floor, has resulted in a noise reduction of $5 \mathrm{~dB}$.

All in all, it would be feasible to think of drawing a process for any built-up structure, which could start with the identification of the most dominant modal transmission paths from source to receiver subsystems, using the graph theory approach depicted heretofore. An analysis could then be made of the mode shapes having the most significant participation in noise transmission (like modes $f_{22}$ and $f_{23}$ ), and then one could try to reduce their energy contribution at the receiver by increasing their damping. A target noise reduction value could be fixed for the receiver (like the $5 \mathrm{~dB}$ in room 6) and appropriate values for the problematic mode damping (like 0.1 for $f_{22}$ and $f_{23}$ ) could be derived to reach that goal. Damping layers could judiciously be placed to reach the objective and determined according to the MSE method. A final SmEdA simulation would verify the performance of the solution in terms of noise reduction. A similar automatized procedure in the easier case of SEA was developed in the framework of graph theory in [28]. The development of an analogous process for SmEdA as described is, however, outside the scope of this paper.

\subsection{Comparison with SEA results}

A comparison with the results that can be obtained from a SEA model of the shipbuilding structure instead of the SmEdA one will be finally presented to highlight some of the advantages of the latter. A SEA

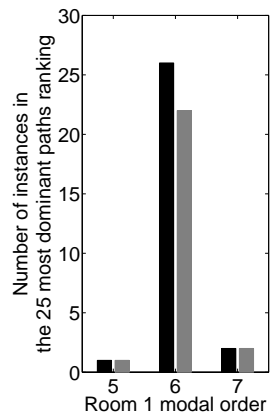

(a)

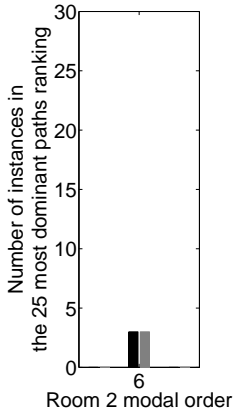

(b)

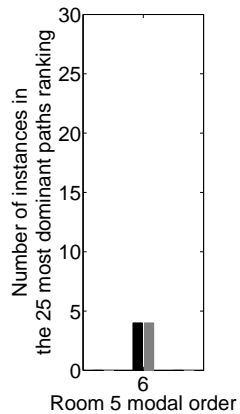

(c)

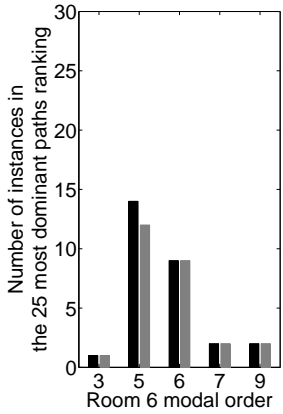

(d)

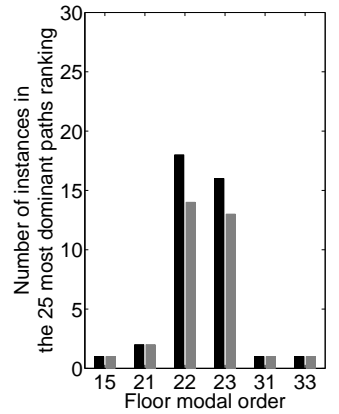

(e)

Figure 14: Number of instances of every mode in the 25 most dominant paths ranking. Black columns: total number of instances in the path ranking. Grey columns: number of paths containing a particular mode. 


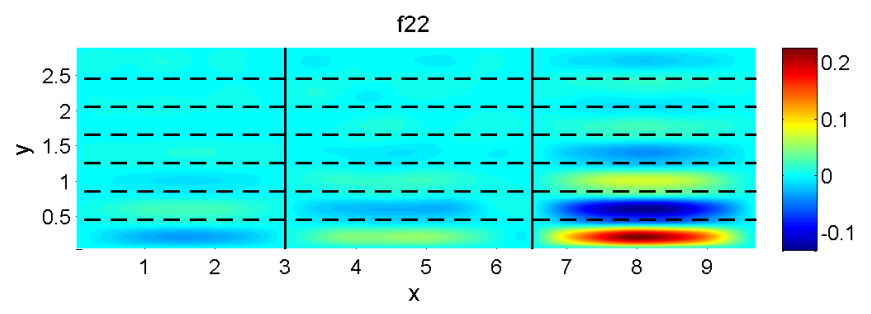

(a)

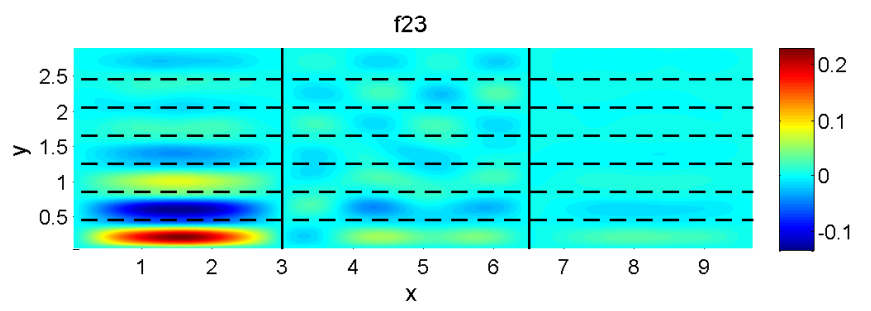

(b)

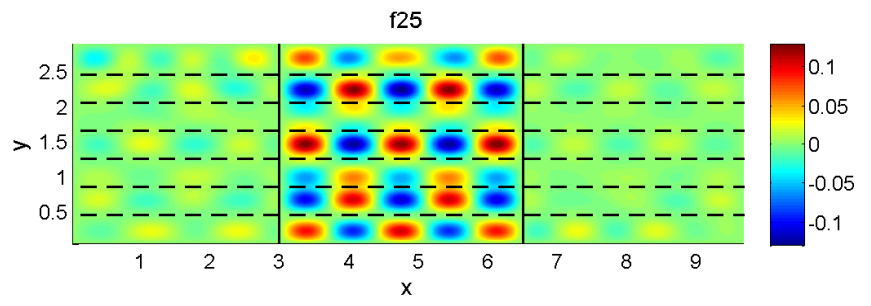

(c)

Figure 15: Magnitudes of the mode spatial shapes on the coupling surface (Horizontal dashed lines: rib positions): (a) $f_{22}$; (b) $f_{23}$; (c) $f_{25}$.

system can be directly deduced from a SmEdA one by assuming modal energy equipartition, see [18, 20]. The SEA coupling loss factors (CLF) and injected power can be related, respectively, to the SmEdA modal coupling factors and modal injected powers. For the basic case of two coupled subsystems considered in Section 2.1, the CLF between subsystems 1 and $2, \eta_{12}$, can be computed as

$$
\eta_{12}=\frac{\sum_{p \in \hat{P}} \sum_{q \in \hat{Q}} \beta_{p q}}{\omega_{c} N_{p}}
$$

and the injected power in subsystem 1 as

$$
\Pi^{1}=\sum_{p \in \hat{P}} \Pi_{i n j}^{p} .
$$

These expressions can be easily generalized in the case of having more subsystems.

When the subsystem modal overlap is high (typically greater than one), energy equipartition takes place to a good extent so that SEA and SmEdA provide similar results. However, for low modal overlap, modal energy equipartition is no longer satisfied for subsystems located far away from the excited one, which leads SEA to overestimate energy transmission [20]. This can be observed in Figs. 13(a) and (b) for the ENR values between rooms 1 and 6 and rooms 1 and 3. In the case of adjacent rooms SEA and SmEdA yield fairly close results. As opposed to this, in the case of rooms 1 and 6 which are at considerable distance apart (see Fig. 11) a strong discrepancy can be observed between the predictions of SmEdA and SEA. The latter gives a lower ENR value and thus a higher energy level at the receiver room 6 . 


\begin{tabular}{|c|l|}
\hline Path & \multicolumn{1}{|c|}{ Shipbuilding SEA } \\
\hline 1 & $S \rightarrow r 1 \rightarrow f \rightarrow r 6 \rightarrow T$ \\
2 & $S \rightarrow r 1 \rightarrow f \rightarrow r 4 \rightarrow r 6 \rightarrow T$ \\
3 & $S \rightarrow r 1 \rightarrow w 2 \rightarrow f \rightarrow r 6 \rightarrow T$ \\
4 & $S \rightarrow r 1 \rightarrow r 3 \rightarrow f \rightarrow r 6 \rightarrow T$ \\
5 & $S \rightarrow r 1 \rightarrow f \rightarrow w 3 \rightarrow r 6 \rightarrow T$ \\
6 & $S \rightarrow r 1 \rightarrow f \rightarrow r 4 \rightarrow f \rightarrow r 6 \rightarrow T$ \\
7 & $S \rightarrow r 1 \rightarrow f \rightarrow r 3 \rightarrow f \rightarrow r 6 \rightarrow T$ \\
8 & $S \rightarrow r 1 \rightarrow r 2 \rightarrow f \rightarrow r 6 \rightarrow T$ \\
9 & $S \rightarrow r 1 \rightarrow f \rightarrow r 5 \rightarrow r 6 \rightarrow T$ \\
10 & $S \rightarrow r 1 \rightarrow f \rightarrow r 6 \rightarrow f \rightarrow r 6 \rightarrow T$ \\
\hline
\end{tabular}

Table 8: Shipbuilding structure example. Ranking of the 10 most dominant energy SEA paths from a total of 100 computed paths for the $200 \mathrm{~Hz} 1 / 3$ octave band.

In Table 8, the ranking for the 10 dominant energy transmission paths between rooms 1 and 6 in the SEA model is presented. As observed the first path clearly involves energy transmission through the floor. However, and contrary to the results in Table 7 , in this case no information at all is available on which modes are responsible for this transmission.

\section{Conclusions}

Energy interchange between modes in subsystems rather than between subsystems (groups of modes) themselves, it is at the very core of SmEdA. As seen, avoiding modal energy equipartition allows one to extend classical SEA to the mid frequency range and dealing with low modal overlap and/or locally excited subsystems, as well as with complex geometry subsystems. However, given that many modes are considered within subsystems, the dimensions of SmEdA matrices become considerably large even for medium sized systems, like those representing sound transmission between two adjacent cavities. Energy transmission in such type of systems is more often caused not so much by mode frequency matching as by mode spatial matching. If one aims at vibroacoustic remedial actions, identifying those resonant and non-resonant modes that play an essential role in energy transmission becomes a must. However, direct mode by mode inspection may become a never-ending task even in the occurrence of frequency matching, as shown for some of the analyzed examples.

It has been proved that graph theory offers a way out to this problem. Analogously to what was previously done in SEA, and for some energy distribution models as well, it becomes possible to define a SmEdA graph associated to any SmEdA model and apply a ranking path algorithm to it. The latter results in a sorted list of modal energy transmission paths from source to receiver subsystems, which allows for the straightforward identification of the modes that dominate the transmission process. Then one could focus on analyzing the spatial matching of the modes in that dominating paths and see whether an increase of damping, a structure reinforcement, or whatever action deemed appropriate, may result in a decrease of the noise transmitted to the receiver. The validity of the graph approach to ease the analysis of SmEdA systems has been demonstrated for different examples, consisting of the transmission of sound between cavities separated by both bare and ribbed plates, as well as for the more complex case of a shipbuilding structure.

\begin{tabular}{|c|c|c|c|c|c|c|c|c|c|c|c|c|c|}
\hline \multirow{2}{*}{$\begin{array}{c}\text { Number } \\
\text { of paths }\end{array}$} & $\begin{array}{c}\text { Accum. } \\
\text { Contr. } \\
\text { [dB] }\end{array}$ & $\begin{array}{c}\text { Accum. } \\
\text { Contr. } \\
\text { [\%] }\end{array}$ & R1 & R2 & R3 & R4 & R5 & R6 & F & W1 & W2 & W3 & W4 \\
\hline$\infty$ & 49.73 & 100.00 & $\checkmark$ & $\checkmark$ & $\checkmark$ & $\checkmark$ & $\checkmark$ & $\checkmark$ & $\checkmark$ & $\checkmark$ & $\checkmark$ & $\checkmark$ & $\checkmark$ \\
100 & 49.73 & 99.93 & $\checkmark$ & $\checkmark$ & $\checkmark$ & $\checkmark$ & $\checkmark$ & $\checkmark$ & $\checkmark$ & $\checkmark$ & $\checkmark$ & $\checkmark$ & $\checkmark$ \\
20 & 49.72 & 99.73 & $\checkmark$ & $\checkmark$ & $\checkmark$ & $\checkmark$ & $\checkmark$ & $\checkmark$ & $\checkmark$ & $\times$ & $\checkmark$ & $\checkmark$ & $\times$ \\
10 & 49.68 & 98.72 & $\checkmark$ & $\checkmark$ & $\checkmark$ & $\checkmark$ & $\checkmark$ & $\checkmark$ & $\checkmark$ & $\times$ & $\checkmark$ & $\checkmark$ & $\times$ \\
1 & 49.47 & 94.12 & $\checkmark$ & $\times$ & $\times$ & $\times$ & $\times$ & $\checkmark$ & $\checkmark$ & $\times$ & $\times$ & $\times$ & $\times$ \\
\hline
\end{tabular}

Table 9: Shipbuilding structure. Transmission path analysis in the SEA model. 


\section{Acknowledgments}

This work has been partially supported by the Labex CeLyA of Université de Lyon, operated by the French National Research Agency (ANR-10-LABX-0060/ANR-11-IDEX-0007). In particular, the short stay of the first author at INSA-Lyon has been funded by this support. The first author would also like to acknowledge the Generalitat de Catalunya (SUR/ECO) for the pre-doctoral FI grant No. 2014FI_B2 00141.

\section{References}

[1] A. Le Bot and V. Cotoni. Validity diagrams of statistical energy analysis. Journal of Sound and Vibration, 329:221-235, 2010

[2] Elke Deckers, Onur Atak, Laurens Coox, Roberto DAmico, Hendrik Devriendt, Stijn Jonckheere, Kunmo Koo, Bert Pluymers, Dirk Vandepitte, and Wim Desmet. The wave based method: An overview of 15 years of research. Wave Motion, 51(4):550 - 565, 2014. Innovations in Wave Modelling.

[3] S. De Rosa and F. Franco. A scaling procedure for the response of an isolated system with high modal overlap factor. Mechanical Systems and Signal Processing, 22(7):1549 - 1565, 2008.

[4] P. Bremner and R.S. Langley. An energy method for midfrequency and low modal overlap. The Journal of the Acoustical Society of America, 100(4):2754-2754, 1996.

[5] R.S. Langley and V. Cotoni. Response variance prediction in the statistical energy analysis of built-up systems. Journal of the Acoustical Society of America, 115:706-718, 2004.

[6] P.J. Shorter and R.S. Langley. On the reciprocity relationship between direct field radiation and diffuse reverberant loading. Journal of the Acoustical Society of America, 117(1):85-95, 2005.

[7] V. Cotoni and P.J. Shorter. Numerical and experimental validation of a hybrid finite element-statistical energy analysis method. Journal of the Acoustical Society of America, 122:259-270, 2007.

[8] Y.H. Park and S.Y. Hong. Hybrid power flow analysis using coupling loss factors of sea for low-damping system - Part I: Formulation of 1-D and 2-D cases. Journal of Sound and Vibration, 299:484-503, 2007.

[9] Y.H. Park and S.Y. Hong. Hybrid power flow analysis using coupling loss factors of sea for low-damping system - Part II: Formulation of 3-D case and PFFEM. Journal of Sound and Vibration, 299:460-483, 2007.

[10] R.S. Langley. A wave intensity technique for the analysis of high frequency vibration. Journal of Sound and Vibration, 159:485-502, 1992.

[11] R.S. Langley and A.N. Bercin. Wave intensity analysis of high frequency vibrations. Philosophical Transactions of the Royal Society A: Physical, Mathematical and Engineering Sciences, 346:489-499, 1994.

[12] A. Le Bot. Energy transfer for high frequencies in built-up structures. Journal of Sound and Vibration, 250 (2):247-275, 2002.

[13] G. Tanner. Dynamical energy analysis - determining wave energy distributions in vibro-acoustical structures in the high-frequency regime. Journal of Sound and Vibration, 320(45):1023 - 1038, 2009.

[14] J.L. Guyader, C. Boisson, and C. Lesueur. Energy transmission in finite coupled plates, part I: theory. Journal of Sound and Vibration, 81(1):81-92, 1982.

[15] C.H. Hodges, P. Nash and J. Woodhouse. Measurement of coupling loss factors by matrix fitting: an investigation of numerical procedures. Applied Acoustics, 22:47-69, 1987.

[16] B.R. Mace. Statistical energy analysis, energy distribution models and system modes. Journal of Sound and Vibration, 275:391-409, 2003.

[17] B.R. Mace. Statistical energy analysis: coupling loss factors, indirect coupling and system modes. Journal of Sound and Vibration, 279:141-170, 2005 .

[18] L. Maxit and J.L. Guyader. Estimation of SEA coupling loss factors using a dual formulations and FEM modal information - Part I: Theory. Journal of Sound and Vibration, 239(5):907 - 930, 2001.

[19] L. Maxit and J.L. Guyader. Estimation of SEA coupling loss factors using a dual formulations and FEM modal information - Part II: Numerical applications. Journal of Sound and Vibration, 239(5):931 - 948, 2001.

[20] L. Maxit and J.L. Guyader. Extension of SEA model to subsystems with non-uniform modal energy distribution. Journal of Sound and Vibration, 265(2):337 - 358, 2003.

[21] N. Totaro and J.L. Guyader. Extension of the statistical modal energy distribution analysis for estimating energy density in coupled subsystems. Journal of Sound and Vibration, 331(13):3114 - 3129, 2012.

[22] L. Maxit, K. Ege, N. Totaro, and J.L. Guyader. Non resonant transmission modelling with statistical modal energy distribution analysis. Journal of Sound and Vibration, 333(2):499 - 519, 2014.

[23] O. Guasch and L. Cortés. Graph theory applied to noise and vibration control in statistical energy analysis models. Journal of the Acoustical Society of America, 125 (6):3657-3672, 2009.

[24] O. Guasch and À. Aragonès. Finding the dominant energy transmission paths in statistical energy analysis. Journal of Sound and Vibration, 330(10):2325 - 2338, 2011.

[25] E.Q.V. Martins. Deviation algorithms for ranking shortest paths. European Journal of Operational Research, 18:123-130, 1984.

[26] E.Q.V. Martins, M.M.B. Pascoal, and J.L.E. Santos. Deviation algorithms for ranking shortest paths. International Journal of Foundations of Computer Science, 10(3):247-261, 1999. 
[27] À. Aragonès and O. Guasch. Ranking paths in statistical energy analysis models with non-deterministic loss factors. Mechanical Systems and Signal Processing, 52 - 53:741 - 753, 2015.

[28] O. Guasch, À. Aragonès, and M. Janer. A graph cut strategy for transmission path problems in statistical energy analysis. Mechanical Systems and Signal Processing, 30:343-355, 2011.

[29] À. Aragonès and O. Guasch. Conditions for transmission path analysis in energy distribution models. Mechanical Systems and Signal Processing, Submitted, 2015.

[30] L. Maxit. Extension et reformulation du modèle SEA par la prise en compte de la répartition des énergies modales (Extension and reformulation of SEA models considering the modal energy distribution). Ph.D. Thesis 2000 INSAL 0016, Institut National des Sciences Appliquées de Lyon, March 2000.

[31] R.H. Lyon and R.G. DeJong. Theory and Application of Statistical Energy Analysis. RH Lyon Corp, Cambridge MA, 2nd Edition, 1998.

[32] R.J.M Craik. Sound Transmission Trough Buildings Using Statistical Energy Analysis. Gower, London, 1996.

[33] C. Hopkins. Sound Insulation. Butterworth-Heinemann, 2007.

[34] R.J.M. Craik. Sound transmission paths through a Statistical Energy Analysis model. Applied Acoustics, 30:45-55, 1990.

[35] O. Guasch. A direct transmissibility formulation for experimental statistical energy analysis with no input power measurements. Journal of Sound and Vibration, 330 (25):6223-6236, 2011.

[36] F.X. Magrans. Method of measuring transmission paths. Journal of Sound and Vibration, 74 (3):311-330, 1981.

[37] O. Guasch and F.X. Magrans. The Global Transfer Direct Transfer method applied to a finite simply supported elastic beam. Journal of Sound and Vibration, 276 (1-2):335-359, 2004.

[38] O. Guasch. Direct transfer functions and path blocking in a discrete mechanical system. Journal of Sound and Vibration, $321(3-5): 854-874,2009$.

[39] R.S. Varga. Matrix iterative analysis, volume 1. Prentice Hall Series in Automatic Computations, Englewood Cliffs: Prentice-Hall, 1962.

[40] F. X. Magrans. Definition and calculation of transmission paths within a SEA framework. Journal of Sound and Vibration, $165(2): 277-283,1993$

[41] B.A. Carré. Graphs and Networks. Oxford Applied Mathematics and Computing Science Series, Oxford University Press, Oxford, 1979

[42] D. Eppstein. Finding the k shortest paths. SIAM Journal on Computing, 28:652-673, 1998.

[43] E. Balmes, J. Bianchi, and J. Leclère. Structural dynamics toolbox \& FEMLink, User's guide. SDTools, Vibration Software and Consulting, 2011.

[44] P. Hynna, P. Klinge, and J. Vuoksinen. Prediction of structure-borne sound transmission in large welded ship structures using statistical energy analysis. Journal of sound and vibration, 180(4):583-607, 1995.

[45] A.C. Nilsson. Attenuation of structure-borne sound in superstructures on ships. Journal of Sound and Vibration, 55(1):7191, 1977.

[46] J. Tratch. Vibration transmission through machinery foundation and ship bottom structure. Master's thesis, Dep. Of Mech. Eng., Massachusetts Institute of Technology, 1985.

[47] R.H. Lyon. In-plane contribution to structural noise transmission. Noise Control Engineering Journal, 26(1):22-27, 1986.

[48] N. Kumar and S.P. Singh. Experimental study on vibration and damping of curved panel treated with constrained viscoelastic layer. Composite Structures, 92(2):233 - 243, 2010.

[49] E.E. Ungar and E.M. Kerwin Jr. Loss factors of viscoelastic systems in terms of energy concepts. The Journal of the acoustical Society of America, 34(7):954-957, 1962.

[50] H. Koruk and K.Y. Sanliturk. Assesment of the complex eigenvalue and the modal strain energy methods for damping predictions. In Proceedings of 18th International Congress on Sound and Vibration, Rio De Janerio, Brazil, 2011.

[51] H. D. Hwang, K. Ege, L. Maxit, N. Totaro, and J.L. Guyader. Equivalent damping modeling in the framework of SmEdA. In Proceedings of XIX-th symposium VIbrations, SHocks \& NOise (VISHNO), Aix en Provence, France, June 2014. 